\title{
High-temperature criticality in strongly constrained quantum systems
}

\author{
Claudio Castelnovo, ${ }^{1}$ Claudio Chamon, ${ }^{1}$ Christopher Mudry, ${ }^{2}$ and Pierre Pujol ${ }^{3}$ \\ ${ }^{1}$ Physics Department, Boston University, Boston, Massachusetts 02215, USA \\ ${ }^{2}$ Paul Scherrer Institut, CH-5232 Villigen PSI, Switzerland \\ ${ }^{3}$ Laboratoire de Physique de l'École Normale Supérieure, Lyon, France
}

(Received 14 December 2005; published 10 April 2006)

\begin{abstract}
The exotic nature of many strongly correlated materials at reasonably high temperatures-for instance, cuprate superconductors in their normal state-has led to the suggestion that such behavior occurs within a quantum-critical region where the physics is controlled by the influence of a phase transition down at zero temperature. Such a scenario can be thought of as a bottom-up approach, with the zero-temperature mechanisms finding a way to manifest critical behavior at high temperatures. Here we propose an alternative, top-down, mechanism by which strong kinematic constraints that can only be broken at extremely high temperatures are responsible for critical behavior at intermediate but still high temperatures. This critical behavior may extend all the way down to zero temperature, but this outcome is not one of necessity, and the system may order at low temperatures. We provide explicit examples of such high-temperature criticality when additional strong interactions are introduced in quantum Heisenberg, transverse-field Ising, and some bosonic lattice models.
\end{abstract}

DOI: 10.1103/PhysRevB.73.144411

PACS number(s): 75.40.-s, 64.60.Fr, 74.20.Mn

\section{INTRODUCTION}

Strongly correlated systems display very rich phase diagrams upon varying thermodynamic parameters such as temperature, pressure, doping concentration, magnetic field, and so on. ${ }^{1}$ At sufficiently low temperatures, distinct phases of matter appear that are characterized by (sometimes coexisting) long-range order of, say, the magnetic, charge, orbital, or superconducting type. When transitions between different zero-temperature quantum phases are continuous, fluctuations occur at all length scales and lead to power-law behavior for correlation functions of the order parameter at the critical coupling. Tuning the temperature slightly away from zero decreases the strength of the order parameter fluctuations but as long as they remain sufficiently strong they lead to scaling laws that are insensitive to the microscopic details and, to a large degree, universality has emerged.

The temperature range for which scaling laws apply is a measure of how strong fluctuations are. In strongly correlated systems such as organic materials, high- $T_{\mathrm{c}}$ superconductors, etc., these scaling laws extend to surprisingly high temperatures above the critical temperature. This fact is attributed to the presence of very strong one- or twodimensional fluctuations of the order parameter that are predominantly quantum at sufficiently short length scales. How large the temperature is at which quantum fluctuations are effectively observed is a matter of intense debate. ${ }^{2}$ For example, it has been proposed that many exotic properties in the so-called pseudogap regime of high- $T_{\mathrm{c}}$ superconductors originate in a hidden quantum critical point. ${ }^{3-5}$ In this scenario, increasing the doping concentration induces at zero temperature a continuous phase transition between two different states of matter below the superconducting dome, which is reflected in the strange metallic properties of the normal state above the superconducting dome.

In this paper, we present an alternative picture where the critical behavior in some strongly interacting systems is present at high temperatures due to strong kinematic constraints in the quantum Hamiltonian. The system remains critical as long as the constraints are respected-i.e., below some large energy scale corresponding to the largest coupling in the Hamiltonian. This critical phase becomes the physically important, universal feature from which a scaling regime can descend. The zero-temperature physics is instead system specific, with a rich variety of different ordered phases, and may or may not allow for the finite-temperature criticality to survive all the way to zero temperature. We also discuss how this picture brings about four distinct scenarios for the finite-temperature behavior of the system, depending on the type of interactions present in the quantum Hamiltonian. In particular, we show how one scenario naturally leads to a quantum system exhibiting an exotic correlation length that increases with increasing temperature over a wide range of temperatures.

The making of a system displaying high-temperature criticality is as follows.

(1) At least one characteristic energy scale in the quantum Hamiltonian - say, $U$-is much larger than all others. Thus, $U$ splits the Hilbert space in sectors separated by large energy gaps. In particular, the infinite- $U$ limit projects the Hilbert space onto the space $\mathcal{H}_{0, U}$ of allowed states.

(2) The quantum dynamics is generated by terms that do not commute with the $U$ term in the quantum Hamiltonian. However, one needs a process of order $n \geqslant 2$ in the perturbative expansion in the characteristic energy scale of these terms, $\Gamma$, to generate an effective quantum coupling between allowed states. Hence, this effective quantum coupling $\Gamma_{\text {eff }} \sim \Gamma(\Gamma / U)^{n-1}$ can be very small, $\left|\Gamma_{\text {eff }}\right| \ll|\Gamma|$.

(3) By squashing down the effective quantum coupling $\Gamma_{\text {eff }}$, one opens a hierarchy of temperature scales, which we discuss in detail in the paper. In particular, already at reasonably low temperatures (above the small $\left.\mid \Gamma_{\text {eff }}\right)$, the effective quantum term can be neglected in the calculation of equilibrium thermodynamic quantities. 
(4) Although the equilibrium thermodynamics for $T \gg\left|\Gamma_{\text {eff }}\right|$ is classical, the dynamics is still quantum. The reason is the following. If the system were to rely on thermally activated processes to move within the restricted Hilbert space $\mathcal{H}_{0, U}$, the characteristic time scale would be, for a small system-bath coupling $\gamma>0, \tau_{T}=\gamma^{-1} \exp (U / T)$, which is astronomical for temperatures well below the large energy scale $U$. There are also virtual processes due to system-bath coupling that bypass thermal activation, but whose effective coupling is suppressed, $\gamma_{\text {eff }} \sim \gamma(\gamma / U)^{n-1} \ll \gamma$, much alike the intrinsic terms that do not commute with the $U$ term. The waiting time for a quantum tunneling event is $\tau_{Q}=\min \left(\left|\Gamma_{\text {eff }}^{-1}\right|, \gamma_{\text {eff }}^{-1}\right)$, which depends only algebraically on $U$. Since $\gamma \ll|\Gamma|$ (the bath coupling should be the weakest term in order not to perturb the energy levels of the system), the intrinsic quantum dynamics provides the smallest dynamical time scale. Hence, a phantom of quantum mechanics in the form of sporadic tunneling events between which coherence is lost provides the fastest mechanism for the system to reach classical thermodynamic equilibrium when $\left|\Gamma_{\text {eff }}\right| \ll T \ll U$.

(5) The strong constraint on the allowed states imposed by taking the limit $U \rightarrow \infty$ first makes the system critical in the constrained entropic limit $T \rightarrow \infty$ with all the remaining characteristic energy scales held fixed. We call such a critical point a constrained entropic critical point. The system's properties at low temperatures compared to $U$ are controlled by the close proximity to this purely constrained entropic critical point. Ice, coloring, and dimer models provide examples of constrained entropic critical points in systems with hard constraints.

(6) Finally, if there are other terms in the Hamiltonian with characteristic energy scale $g,|g| \ll U$, but that commute with the $U$ term, there can be different phases and transitions among them when $T /|g|$ is $\mathcal{O}(1)$. When $U \gg T \gg|g|$, the physics is controlled by the proximity to the constrained entropic critical point. Even though the constrained entropic critical point may be unstable, the nearby renormalization group (RG) trajectories feel its presence until the RG scale $T \sim U$ is reached, beyond which the constraint becomes immaterial. The featureless unconstrained (stable) fixed point takes over at that stage.

The phase diagram in Fig. 1 exemplifies the hightemperature critical behavior arising from kinematic constraints that we highlight in this paper. It shows a large region-the constrained entropic critical regime-standing in between two ordered phases. The constrained entropic critical region exists because of the strong constraints imposed by the large energy scale $U$ in the problem, and it covers a high-temperature range that ends at the extreme limit of temperatures of order $U$, where the system becomes featureless in that it is controlled by its proximity to the unconstrained entropic fixed point (paramagnetic phase). The ordered phases to the left or right of the constrained entropic critical regime have a classical origin in Fig. 1, since there the second largest energy scale $|g|$ is associated with an operator that commutes with the constraint. If we fix $\Gamma_{\text {eff }}$ and vary $g$, as in Fig. 1, the high-temperature constrained entropic critical region sits on top of a quantum phase with radius of the order of $\left|\Gamma_{\text {eff }}\right|$ around the origin $T=g=0$. Quantum criticality may exist only within this small region.

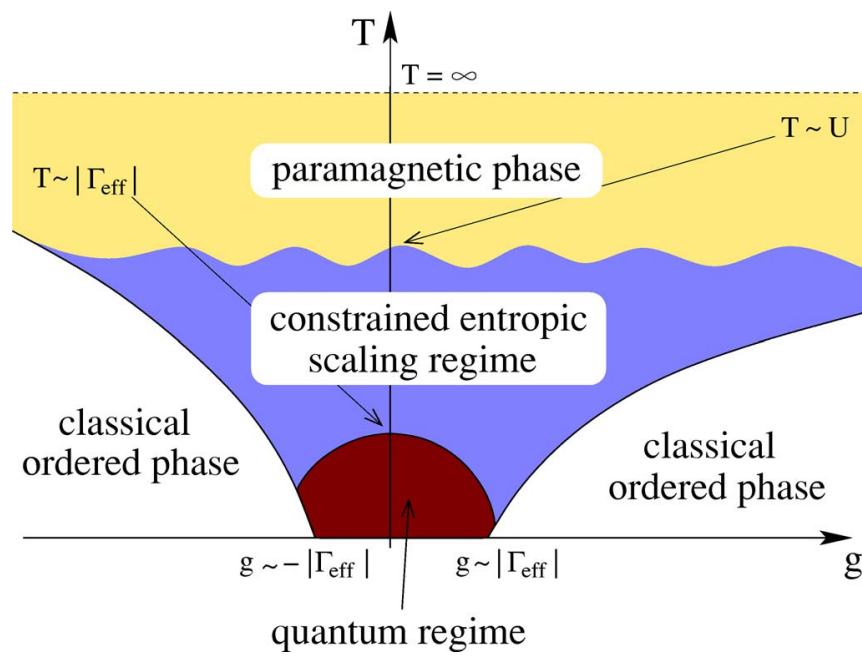

FIG. 1. (Color online) Generic phase diagram for a strongly constrained quantum system that satisfies conditions (1)-(6) in Sec. I. The parameter space encodes the competition between two energy scales, the temperature $T$, and a characteristic energy scale $g$ that selects a classical ordered phase, while $\Gamma_{\text {eff }}$ and $U$ are held fixed. A quantum-critical scaling regime, if it exists, is restricted to a region represented by the upper half of a disk of radius $\sim\left|\Gamma_{\text {eff }}\right|$ and centered at the origin $(g, T)=(0,0)$ of parameter space. The focus of this paper is on the constrained entropic scaling regime at temperatures intermediate between the small characteristic quantum energy scale $\Gamma_{\text {eff }}$ and the large characteristic energy scale $U$ set by a strong constraint. At a fixed temperature, the constrained entropic scaling regime terminates in a phase transition that needs not be continuous upon increasing $|g|$. At fixed $g$, the constrained entropic scaling regime crosses over to the conventional high-temperature phasesay, a paramagnetic one for spin degrees of freedom-when $T$ is of the order of $U$. The transition from the constrained entropic scaling regime to the quantum regime upon lowering $T$ at fixed $g$ is system specific.

We illustrate this constraint-based critical behavior at high temperatures using simple case studies, a constrained quantum Heisenberg model and a constrained Ising model in a transverse field both on the honeycomb lattice, and a constrained bosonic model on the square lattice that leads to the quantum dimer model at low energies. The single-band Hubbard model, where a large on-site repulsive term $U$ is the dominant energy scale, fails to fulfill condition (5), and correlations decay exponentially fast with separation beyond a characteristic length scale of the order of the lattice spacing. However, this situation may change if one considers extended Hubbard models with large nearest-neighbor coupling $V$, next-nearest-neighbor coupling $V^{\prime}$, etc., at commensurate fillings. We shall comment on this situation in the Conclusions and discuss, for instance, the possible connection between the ideas of constrained entropic criticality and those of fluctuating stripes. ${ }^{5}$ In addition, power-law behavior at high temperatures was also shown to occur in the context of locally fluctuating bond currents in $d$-density-wave states by Chakravarty in Ref. 6, who also recognized the importance of the constraints in determining the long-distance correlations in the system.

The plan of the paper is as follows. Four examples of constrained quantum models are introduced in Sec. II. The 
regimes of temperature that are of relevance to this paper are presented in Sec. III. The constrained entropic scaling regime is described in Sec. IV. Realizations of constrained entropic scaling regimes for quantum $X X Z$ Heisenberg and transverse-field Ising models, on the one hand, and lattice bosonic models, on the other hand, can be found in Secs. V and VI, respectively. In Sec. VII we discuss the regime when the quantum scale $\left|\Gamma_{\text {eff }}\right|$ is the largest scale below $U$-i.e., $|g| \ll\left|\Gamma_{\text {eff }}\right| \ll U$ - and we conclude in Sec. VIII.

\section{FOUR EXAMPLES OF CONSTRAINED QUANTUM MODELS}

Constrained quantum-mechanical systems are described by Hamiltonians of the generic form

$$
\begin{aligned}
& \hat{H}=\hat{H}_{g, \Gamma}+\hat{H}_{U}, \\
& \hat{H}_{g, \Gamma}=\hat{H}_{g}+\hat{H}_{\Gamma},
\end{aligned}
$$

where it is assumed that $\hat{H}_{U}$ can be diagonalized in some preferred basis $\mathcal{B}$ that spans the Hilbert space $\mathcal{H}$ on which $\hat{H}$ is defined, $\hat{H}_{g}$ commutes with $\hat{H}_{U}$,

$$
\left[\hat{H}_{g}, \hat{H}_{U}\right]=0,
$$

$\hat{H}_{\Gamma}$ does not commute with $H_{U}$,

$$
\left[\hat{H}_{\Gamma}, \hat{H}_{U}\right] \neq 0,
$$

and the characteristic energy scale $U$ of $\hat{H}_{U}$ is much larger than the characteristic energy scales $g$ and $\Gamma$ of $\hat{H}_{g}$ and $\hat{H}_{\Gamma}$, respectively,

$$
|g|, \quad|\Gamma| \ll U .
$$

A famous example of a constrained many-body Hamiltonian is the single-band Hubbard model ${ }^{7}$

$$
\begin{aligned}
\hat{H}:= & -\mu \sum_{i} \sum_{\sigma=\uparrow, \downarrow} \hat{c}_{i \sigma}^{\dagger} \hat{c}_{i \sigma} \\
& -t \sum_{\langle i j\rangle} \sum_{\sigma=\uparrow, \downarrow}\left(\hat{c}_{i \sigma}^{\dagger} \hat{c}_{j \sigma}+\hat{c}_{j \sigma}^{\dagger} \hat{c}_{i \sigma}\right) \\
& +U \sum_{i} \prod_{\sigma=\uparrow, \downarrow}\left(\hat{c}_{i \sigma}^{\dagger} \hat{c}_{i \sigma}\right),
\end{aligned}
$$

which acts on the fermionic Fock space generated by the creation $\hat{c}_{i \sigma}^{\dagger}$ and annihilation $\hat{c}_{i \sigma}$ operators for electrons carrying the site index $i$ and the spin index $\sigma$. The preferred basis $\mathcal{B}$ is the basis specified by all local fermionic occupation numbers $\hat{c}_{i \sigma}^{\dagger} \hat{c}_{i \sigma}$ where $i$ runs over all lattice sites and $\sigma$ over the spin up or down. In this preferred basis, the chemical potential (2.2a) and the potential energy (2.2c) are diagonal whereas the kinetic energy (2.2b) is not. The chemical potential $\mu$ thus plays the role of $g$ while the hopping amplitude $t$ plays the role of $\Gamma$.

A second example is the constrained $X X Z$ quantum spin$1 / 2$ magnet defined on the two-dimensional honeycomb lattice by the Hamiltonian ${ }^{8,9}$

$$
\begin{aligned}
\hat{H}:= & -J \sum_{\langle i j\rangle} \hat{\sigma}_{i}^{z} \hat{\sigma}_{j}^{z}-\sum_{i=1}^{N}\left[h+(-1)^{i} h_{s}\right] \hat{\sigma}_{i}^{z} \\
& -\Gamma \sum_{\langle i j\rangle}\left(\hat{\sigma}_{i}^{x} \hat{\sigma}_{j}^{x}+\hat{\sigma}_{i}^{y} \hat{\sigma}_{j}^{y}\right) \\
& +U \sum_{\square}\left[1-\cos \left(2 \pi \sum_{i \in \bigcirc} \hat{\sigma}_{i}^{z} / 3\right)\right],
\end{aligned}
$$

where the sum over the symbol $\square$ is to be understood as a summation over all elementary hexagons making up the honeycomb lattice and the three $2 \times 2$ Pauli matrices are denoted by $\hat{\sigma}^{x}, \hat{\sigma}^{y}, \hat{\sigma}^{z}$, respectively. The preferred basis $\mathcal{B}$ is the basis specified by all the eigenstates with eigenvalues $\sigma_{i}^{z}$ of $\hat{\sigma}_{i}^{z}$, where $i$ runs over all $N$ sites of the honeycomb lattice. In this preferred basis the potential energy (2.3c) and the longitudinal one- and two-body interactions (2.3a) are diagonal whereas the transverse two-body interaction $(2.3 \mathrm{~b})$ is not. The exchange coupling $J$, the uniform magnetic field $h$, and the staggered magnetic field $h_{s}$ thus play the role of three different diagonal couplings $g$.

A third example is the constrained quantum Ising model in a transverse field,,, 10

$$
\begin{aligned}
\hat{H}:= & -J \sum_{\langle i j\rangle} \hat{\sigma}_{i}^{z} \hat{\sigma}_{j}^{z}-\sum_{i=1}^{N}\left[h+(-1)^{i} h_{s}\right] \hat{\sigma}_{i}^{z} \\
& -\Gamma \sum_{i=1}^{N} \hat{\sigma}_{i}^{x} \\
& +U \sum_{\bigcirc}\left[1-\cos \left(2 \pi \sum_{i \in \bigcirc} \hat{\sigma}_{i}^{z} / 3\right)\right],
\end{aligned}
$$

which shares with the spin-1/2 quantum Hamiltonian (2.3) the same preferred basis. In this preferred basis, the potential energy (2.4c) and the one- and two-body interactions (2.4a) are diagonal whereas the one-body transverse field $(2.4 \mathrm{~b})$ is not. The exchange coupling $J$, the uniform magnetic field $h$, and the staggered magnetic field $h_{s}$ thus play again the role of three different couplings $g$.

Our last example is the quantum Hamiltonian

$$
\begin{aligned}
\hat{H}:= & v \sum_{i=1}^{N}\left[\left(\hat{b}_{i, i+x}^{\dagger} \hat{b}_{i, i+x}\right)\left(\hat{b}_{i+y, i+y+x}^{\dagger} \hat{b}_{i+y, i+y+x}\right)+\boldsymbol{x} \leftrightarrow \boldsymbol{y}\right] \\
& -t \sum_{i=1}^{N}\left[\hat{b}_{i, i+x}^{\dagger}\left(\hat{b}_{i, i+y}+\hat{b}_{i+x, i+x+y}+\hat{b}_{i-y, i}+\hat{b}_{i+x-y, i+x}\right)+\text { H.c. }\right] \\
& +U \sum_{i=1}^{N}\left[1-\sum_{e=x, y}\left(\hat{b}_{i-e, i}^{\dagger} \hat{b}_{i-e, i}+\hat{b}_{i, i+e}^{\dagger} \hat{b}_{i, i+e}\right)\right]^{2}
\end{aligned}
$$

which acts on the bosonic Fock space generated by the bosonic creation $\hat{b}_{i, i+e}^{\dagger}$ and annihilation $\hat{b}_{i, i+e}$ operators defined on the midpoints of the nearest-neighbor links of the square lattice. The preferred basis $\mathcal{B}$ is the basis specified by 
all the local bosonic occupation numbers-i.e., the eigenvalues $n_{i, i+e}$ of

$$
\hat{n}_{i, i+e}:=\hat{b}_{i, i+e}^{\dagger} \hat{b}_{i, i+e}
$$

for all $2 N$ links $i, i+\boldsymbol{e}$ where $i$ runs over the $N$ sites of the square lattice and $\boldsymbol{e}$ over its two generating vectors $\boldsymbol{x}$ and $\boldsymbol{y}$. In this preferred basis the potential energy $(2.5 \mathrm{c})$ and the four-body interaction (2.5a) are diagonal whereas the kinetic energy (2.5b) is not. The coupling $v$ thus plays the role of $g$ while the coupling $t$ plays the role of $\Gamma$.

By assumption, the contribution $\hat{H}_{U}$ encodes the largest characteristic energy scale $U$ in the problem. As long as all other energy scales are much smaller than $U$, the low-energy physics is captured by an effective Hamiltonian $\hat{H}_{\text {eff }}$ that is defined on the Hilbert space restricted to the lowest-energy eigenstates of $\hat{H}_{U}$. $\hat{H}_{\text {eff }}$ can be systematically deduced from $\hat{H}$ by treating all the contributions $\hat{H}_{\Gamma}$ to $\hat{H}_{g, \Gamma}$ that do not commute with $\hat{H}_{U}$ within perturbation theory. This effective model is particularly interesting whenever the ground-state manifold $\mathcal{H}_{0, U}$ of $\hat{H}_{U}$ is extensively degenerate, as is the case for all examples (2.2)-(2.5). Within degenerate perturbation theory, $\hat{H}_{\text {eff }}$ is given by

$$
\hat{H}_{\text {eff }}=\hat{H}_{g}-\Gamma_{\text {eff }} \hat{H}_{\Gamma / U}^{(n)},
$$

with

$$
\Gamma_{\text {eff }} \propto \frac{\Gamma^{n}}{U^{n-1}}
$$

and $\hat{H}_{\Gamma / U}^{(n)}$ of order zero in $\Gamma / U$, whereby it is understood that $\hat{H}_{\text {eff }}$ acts only on the subspace $\mathcal{H}_{0, U}$ of the unconstrained Hilbert space with basis $\mathcal{B}$. The order $n$ and the form taken by $\hat{H}_{\Gamma / U}^{(n)}$ in Eq. (2.7a) are model dependent.

For the Hubbard model (2.2), $n=1$ and $^{11-13}$

$$
\begin{aligned}
\hat{H}_{\mathrm{eff}}= & -\mu \sum_{i} \sum_{\sigma=\uparrow, \downarrow} \hat{c}_{i \sigma}^{\dagger} \hat{c}_{i \sigma}-t\left\{\sum_{\langle i j\rangle} \sum_{\sigma=\uparrow, \downarrow}\left(\hat{c}_{i \sigma}^{\dagger} \hat{c}_{j \sigma}+\hat{c}_{j \sigma}^{\dagger} \hat{c}_{i \sigma}\right)\right. \\
& \left.+\frac{4 t}{U} \sum_{\langle i j\rangle}\left(\hat{\boldsymbol{S}}_{i} \cdot \hat{\boldsymbol{S}}_{j}-\frac{1}{4} \hat{n}_{i} \hat{n}_{j}\right)+\cdots\right\}
\end{aligned}
$$

acts on the subspace $\mathcal{H}_{0, U}$ of the fermionic Fock space with no more than one electron per site. Here, we have introduced the fermionic bilinears $\hat{S}_{i}:=\hat{c}_{i \sigma}^{\dagger} \frac{\sigma_{\sigma \sigma^{\prime}}}{2} \hat{c}_{i \sigma^{\prime}}$ and $\hat{n}_{i}:=\hat{c}_{i \sigma}^{\dagger} \hat{c}_{i \sigma}$ (summation over repeated spin indices is implied). Notice that, at half-filling, all contributions to first order in $t$ vanish so that $\Gamma_{\text {eff }}$ becomes second order in $t(n=2)$.

For the constrained $X X Z$ quantum spin-1/2 magnet on the honeycomb lattice (2.3), $n=3$ in Eqs. (2.7) whereby the constrained Hilbert space is the subspace $\mathcal{H}_{0, U}$ defined by all states of the form $\left|\sigma_{1}^{z}, \ldots, \sigma_{N}^{z}\right\rangle$ such that the magnetization of each elementary hexagonal plaquette is some integer multiple of 3 -i.e.,

$$
\sum_{i \in \subseteq} \sigma_{i}^{z} / 3 \in \mathbb{Z}
$$

For the constrained quantum Ising model in a transverse field on the honeycomb lattice (2.4), $n=6$, and the constrained Hilbert space is the same as in example (2.3).

At last, $n=2$ for the bosonic Hamiltonian (2.5) whereby the constrained Hilbert space is the subspace $\mathcal{H}_{0, U}$ spanned by all states of the form $\left|\ldots, n_{\langle i j\rangle}, \ldots\right\rangle$, where $\langle i j\rangle$ runs over all $2 N$ links of the square lattice made of $N$ sites and the boson occupation numbers are restricted to

$$
1=\sum_{\boldsymbol{e}=\boldsymbol{x}, \boldsymbol{y}}\left(n_{i-\boldsymbol{e}, i}+n_{i, i+e}\right)
$$

for all $N$ sites $i$. This condition is satisfied if $n_{\langle i j\rangle}=0,1$ and only one link out of the four connected to each vertex is occupied by a boson. This constrained system is equivalent to a square lattice quantum dimer model.

The last three models will be studied in detail in Secs. V and VI, which the reader is referred to for an example-based approach. In Secs. III and IV we now discuss the possible topologies of the phase diagram when a strongly constrained quantum system possesses a constrained critical regime at intermediary temperatures.

\section{COMPETING CHARACTERISTIC ENERGY SCALES}

From the generic form taken by the low-energy Hamiltonian (2.7) acting on the ground-state manifold $\mathcal{H}_{0, U}$ and from the assumption that $|g|,|\Gamma| \ll U$, we deduce the existence of at least three regimes of temperatures provided any one of the hierarchies of energy scales

$$
\begin{gathered}
T \ll\left|\Gamma_{\text {eff }}\right|, \quad|g| \ll U, \\
\left|\Gamma_{\text {eff }}\right|, \quad|g| \ll T \ll U, \\
\left|\Gamma_{\text {eff }}\right|, \quad|g| \ll U \ll T
\end{gathered}
$$

holds. In addition to these three temperature regimes, strongly constrained systems described by the low-energy Hamiltonian (2.7) can exhibit a fourth regime if $|g|$ is much larger than all effective off-diagonal couplings. This is the case, for example, when $|g| \geq|\Gamma|$ and the off-diagonal terms in $\hat{H}_{\Gamma}$ do not contribute to first order in $\Gamma$-i.e., $n>1$ in Eq. (2.7b). If so, $\left|\Gamma_{\text {eff }}\right|$ is guaranteed to be much smaller than $g$ and the temperature regime (3.1a) can be further subdivided into two distinct ones, for a total of four regimes

$$
\begin{aligned}
& T \ll\left|\Gamma_{\text {eff }}\right| \ll|g| \ll U, \\
& \left|\Gamma_{\text {eff }}\right| \ll T \ll|g| \ll U, \\
& \left|\Gamma_{\text {eff }}\right| \ll|g| \ll T \ll U, \\
& \left|\Gamma_{\text {eff }}\right| \ll|g| \ll U \ll T .
\end{aligned}
$$

For simplicity, we shall consider only two limiting cases that encode the competition between the classical energy 
scale $g$ and the quantum energy scale $\Gamma_{\text {eff }}$ in this paper. The first occurs when $|g| \gg\left|\Gamma_{\text {eff }}\right|$ in the temperature regime (3.2c). The second occurs when $|g| \ll\left|\Gamma_{\text {eff }}\right|$ in the temperature regime (3.1b). Both situations can be realized in examples (2.3)-(2.5). In particular, the case of a quantum energy scale dominating over the classical one is of relevance to the Hubbard model close to half-filling, to quantum $X X Z$ Heisenberg magnets with strong $X Y$ exchange anisotropy, to Ising magnets subjected to strong transverse magnetic fields, or to constrained bosons whose kinetic energy dominates over their interactions. We postpone the discussion of the second case to Sec. VII, while we consider here the case $|g| \gg\left|\Gamma_{\text {eff }}\right|$.

The quantum world at $T=0$ is governed by a delicate competition between the diagonal and off-diagonal energy scales. As these characteristic energy scales are varied, quantum phase transitions can take place between different states of matter that usually support long-range order. Universality emerges at fine-tuned quantum critical points where the transitions between different states of matter are continuous. The signature of a quantum critical point can manifest itself as a scaling regime as long as temperatures are not too largesay, when Eq. (3.2a) holds.

Upon increasing the temperature from $T=0$, one leaves the quantum regime (3.2a) once $T$ becomes larger than the quantum coupling $\left|\Gamma_{\text {eff }}\right|$ in the restricted Hilbert space. Beyond the quantum regime one distinguishes the three regimes (3.2b), (3.2c), and (3.2d). If the temperature is much smaller than $|g|$, the thermal fluctuations are dominated by the classical energy scale $g$. If the temperature is raised to values that are much larger than $|g|$ but that remain much smaller than the characteristic constraint energy $U$, then the thermal fluctuations are predominantly entropic in character, with the entropy of the classical constrained phase space isomorphic to the preferred basis $\mathcal{B}_{0, U}$ that spans the ground-state manifold $\mathcal{H}_{0, U}$ of $\hat{H}_{U}$. Finally, once the temperature becomes the largest energy scale in the problem, the thermal fluctuations are still entropic in character, but now with the entropy of the classical unconstrained phase space isomorphic to the basis $\mathcal{B}$ of $\hat{H}$. The transitions between these regimes can take place through phase transitions or through crossovers.

The two entropic regimes (3.2c) and (3.2d) do not always need to be qualitatively different. This is the case for the Hubbard model for which all connected spatial correlation functions between the local electronic densities $\hat{n}_{i \sigma}=\hat{c}_{i \sigma}^{\dagger} \hat{c}_{i \sigma}$ in the entropic regime (3.2c) decay in a qualitatively similar way as in the entropic regime (3.2d)-i.e., exponentially fast with separation beyond a characteristic length scale of the order of the lattice spacing. The situation may change if one considers extended Hubbard models with nearest-neighbor coupling $V$, next-nearest-neighbor coupling $V^{\prime}$, etc., at commensurate fillings. We shall comment on this situation in the Conclusions. In the examples (2.3)-(2.5), we shall show below that the two entropic regimes (3.2c) and (3.2d) are qualitatively different as measured by the temperature dependence and order of magnitude of the correlation length characterizing the onset of exponential decay in spatial correlation functions.

\section{CONSTRAINED ENTROPIC SCALING REGIME}

In this section we shall study the generic features of the constrained entropic regime (3.2c) for Hamiltonians of the type (2.3)-(2.5). All the assumptions and different cases considered here will be supported with explicit examples in Secs. V and VI. The reader who may be unfamiliar with the physics of constrained models is referred to those two sections for an example-based approach.

Since quantum dynamics is of no qualitative relevance in the regime (3.2c), we shall always assume that $\left|\Gamma_{\text {eff }}\right| / T \rightarrow 0$ while keeping the ratios $|g| / T \ll 1$ and $T / U \ll 1$ fixed. Were it not for the presence of the constraint in the regime (3.2c), set by the large energy scale $U \gg T$, the condition $T \gg|g|$ would place the system deep into a massive phase; i.e., correlation functions would decay exponentially in space with a characteristic decay length, the correlation length, of the order of the lattice spacing $\mathfrak{a}$. We are going to argue that, in the examples (2.3)-(2.5), the constraining energy scale $U$ induces a correlation length much larger than the lattice spacing and possibly increasing with temperature in the regime (3.2c).

Without loss of generality $g>0$ is assumed in the remainder of this section.

\section{A. Scaling limit $g / T, T / U \rightarrow 0$}

We begin our analysis by considering the scaling limit $g / T, T / U \rightarrow 0$. In this limit, all entropic fluctuations are restricted to the classical configuration space isomorphic to the basis $\mathcal{B}_{0, U}$. This hard constraint has dramatic consequences on thermal averages in the examples (2.3)-(2.5). Indeed any spatial spin-spin correlation functions in the examples (2.3) and (2.4) or spatial correlation functions between the local bosonic densities $n_{\langle i j\rangle}$ in the example (2.5) decay algebraically with separation in the scaling regime $g / T, T / U \rightarrow 0$. This is so because the examples (2.3) and (2.4) reduce to the noninteracting classical three-coloring model that was solved by Baxter in Ref. 14, whereas the example (2.5) reduces to the noninteracting classical square-lattice dimer model that was solved by Kasteleyn in Ref. 15. In either case, it is now understood that the constrained entropic scaling limit $g / T, T / U \rightarrow 0$ is critical in that correlation functions decay as power laws in space.

\section{B. Scaling limit $g / T \rightarrow 0,0<T / U \ll 1$}

In this paper, we shall assume that, if we soften the condition that entropic fluctuations satisfy the constraint-i.e., if we consider the limit $g / T \rightarrow 0$ holding $T / U$ small but finite-we must then impose a cutoff $\xi_{\mathrm{ce}}(T / U)$ to the spatial algebraic decay of correlation functions. In effect, we are assuming that the operator related to the appearance of defects that violate the constraint imposed by the energy scale $U$ is a relevant perturbation to the constrained entropic critical point in the RG sense. This assumption is indeed satisfied by the examples (2.3)-(2.5) but there are no fundamental reasons for it to hold for all constrained systems.

If defects cause the system to flow to a generic unconstrained fixed point with short-range correlations in space, the correlation length of the system is controlled by the ratio $T / U$ and can be estimated with the following argument. The concentration of thermally activated defects that violate the constraint is proportional to $\exp \left(-\alpha_{U} U / T\right)$ when $T \ll U$, 
where $\alpha_{U}>0$ is some nonuniversal numerical constant specific to $\hat{H}_{U}$, since it represents the fugacity of defects that enters as the coupling constant driving the system away from the constrained entropic scaling limit. Therefore, the correlation length of the system is

$$
\xi_{\mathrm{ce}}(T / U) \sim \mathfrak{a} \exp \left(\frac{\alpha_{U}}{d-y_{U}} \frac{U}{T}\right),
$$

when $T \ll U$. Here, $\mathfrak{a}$ is the lattice spacing, $d$ is the dimensionality of space, and $0<y_{U}<d$ is the scaling dimension of the operator representing a defect. Upon approaching the constrained entropic critical point $T / U=0$, the exponential growth of the correlation length $\xi_{\mathrm{ce}}(T / U)$ guarantees that correlation functions decay in space as power laws over a macroscopically large window of length scales

$$
\mathfrak{a} \ll r \ll \xi_{\text {ce }}(T / U) .
$$

While the entropic regime (3.2c) of the constrained Hubbard model is featureless beyond few lattice spacings, the entropic regimes (3.2c) of the examples (2.3)-(2.5) exhibit a scaling behavior over an exponentially large window of length scales. For length scales large compared to the lattice spacing but smaller than $\xi_{\mathrm{ce}}(T / U)$ the system behaves as a noninteracting classical system with the constraint fully enforced, whose correlation functions are captured by a (purely entropic) critical theory. As the ratio $T / U$ is increased to a value of order 1 , the correlation length $\xi_{\mathrm{ce}}(T / U)$ decreases until it becomes of the same order of the lattice spacing.

An interesting possibility occurs if the relevant coupling constant that drives the system away from an unstable constrained entropic fixed point generates a correlation length $\xi_{\mathrm{ce}}(T / U)$ that is an algebraic function of $T / U$ as opposed to an exponential one in $T / U$. If so, the correlation length $\xi_{\mathrm{ce}}(T / U) \sim(T / U)^{-\nu_{U}}$ for some correlation length exponent $\nu_{U}$. This scaling of the correlation length with temperature is identical to that near a quantum-critical point, $\xi_{\mathrm{QCP}} \sim T^{-1 / z}$ for some dynamical exponent $z$. It may thus deceive observers expecting quantum-critical scaling: the behavior is classical as we are in the temperature regime (3.2c), very far past the quantum regime (3.2a).

\section{Scaling limit $T / U \rightarrow 0,0<g / T \ll 1$}

We now perturb the constrained entropic critical point $(g / T, T / U)=(0,0)$ by working at a finite value of $g / T$ while $T / U=0$. We call scenario I the case when $g / T$ is irrelevant if sufficiently small but becomes relevant beyond some finite critical value $(g / T)_{\mathrm{c}}$. We call scenario II the case when any finite value of $g / T$ is relevant. Scenario III occurs when $g / T$ is exactly marginal up to some critical value $(g / T)_{\mathrm{c}}$, in which case the segment $0 \leqslant g / T \leqslant(g / T)_{\text {c }}$ realizes a line of critical points. At last, scenario IV happens when the small coupling $g / T$ preserves criticality but strongly alters its nature; for instance, it changes continuously the value of the central charge in the two-dimensional example given in Sec. V D.

In the case of scenario I, all properties of the constrained entropic critical point $(g / T, T / U)=(0,0)$ survive a sufficiently small perturbation $g / T$ until it reaches the critical value $(g / T)_{\mathrm{c}}$ at which a phase transition takes place to a noncritical phase of matter selected by the characteristic energy scale $g$. This classical phase transition can be continuous but need not be so. The noncritical phase of matter could support a conventional classical long-range order such as, say, antiferromagnetic order. If classical frustration effects are prevalent, the noncritical phase of matter could support less conventional classical order such as spin-glass order. An exotic possibility occurs when classical frustration effects select a noncritical phase devoid of any long-range order.

In the case of scenario II, any finite $g / T$ generates a finite correlation length $\xi_{\mathrm{II}}(g / T)$ by causing the system to order into a noncritical phase. This correlation length diverges with $g / T \rightarrow 0$ in the case of a continuous classical phase transition at infinite temperature, but it may as well remain finite-for example, if the transition is first order.

Scenarios III and IV differ from scenario I in that the segment $0 \leqslant g / T \leqslant(g / T)_{\mathrm{c}}$ with $T / U=0$ is a line of critical points in both cases. The number of critical degrees of freedom is unchanged in case III whereas it does change in case IV as a function of $0 \leqslant g / T \leqslant(g / T)_{\text {c }}$.

The constrained quantum $X X Z$ Heisenberg and transverse field Ising models (2.3) and (2.4), respectively, provide explicit realizations of scenarios I, II, III, and IV, as we will demonstrate in Secs. V and VI.

\section{Perturbing the constrained entropic critical point with $\mathbf{0}<\boldsymbol{T} / U$ and $\mathbf{0}<\mathrm{g} / \mathrm{T}$}

The fate of the transition at $\left((g / T)_{c}, 0\right)$ on the boundary of the phase diagram parametrized by the dimensionless couplings $(g / T, T / U)$ is model dependent as one moves to the interior of the phase diagram.

For simplicity, we assume that the phase diagram consists of two competing phases only. One phase is the basin of attraction of the unconstrained entropic stable fixed point located at $(g / T, T / U)=(0, \infty)$. The other phase is the basin of attraction of the stable fixed point located at $(g / T, T / U)$ $=(\infty, 0)$. A cartoon version of this phase diagram is depicted in Fig. 2 (top) for scenarios I, III, or IV and in Fig. 2 (bottom) for scenario II. Curves with the dimensionless ratio $g / U$ held fixed are represented by dashed lines in Fig. 2 . Changing the temperature for some given $g / U$ corresponds to moving along a dashed line in Fig. 2. The smaller the ratio $g / U$, the larger the temperature range for which the system lingers in the vicinity of the constrained entropic critical point $(0,0)$ along a line with $g / U$ held fixed.

Finally, observe that the location of the phase boundary close to the constrained entropic critical point $(0,0)$ follows from

$$
\xi_{\text {II }}(g / T) \sim \xi_{\mathrm{ce}}(T / U)
$$

in the case of scenario II, assuming that the phase boundary is a line of continuous transitions. One interesting feature of scenario II is that the correlation length increases as temperature increases for a large range of temperatures. The reason is that, since the constrained entropic fixed point is unstable in both horizontal $(g / T)$ and vertical $(T / U)$ directions, as the temperature is raised and one moves in parameter space 
(a)

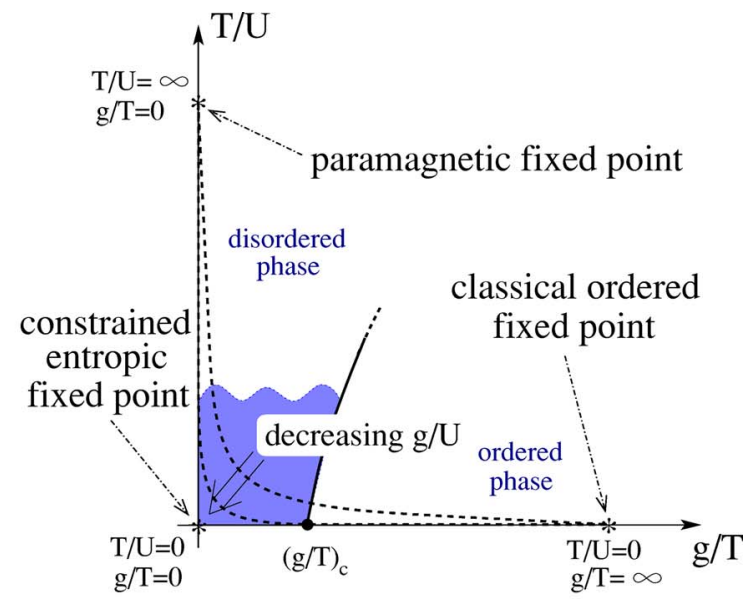

(b)

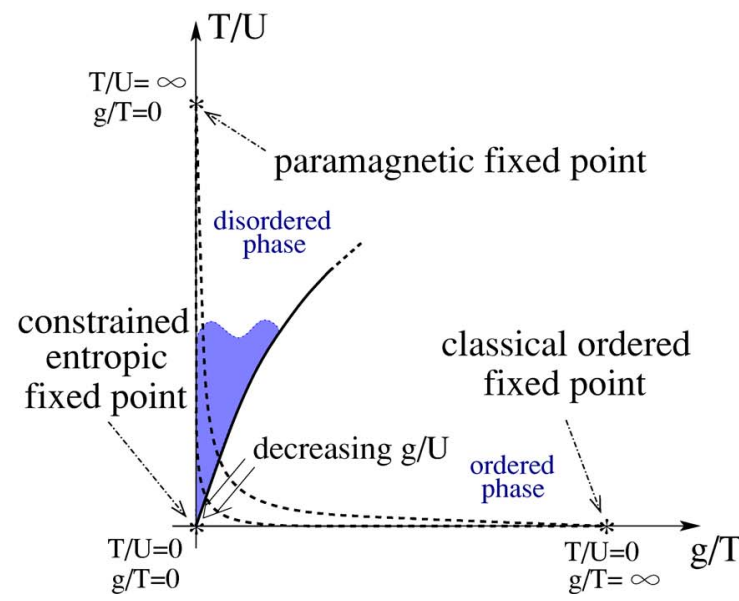

FIG. 2. (Color online) Qualitative phase diagram of the classical constrained system assuming the existence of two phases, a classical ordered phase and a disordered one, separated by a phase boundary that extends all the way to $T=0$. Not all of the phase boundary between the ordered phase and the paramagnetic phase is shown here. The topology of the phase boundary upon approaching $T=0$ depends on the microscopics, as we imply by depicting the small- $T$ side of the phase boundary with a dotted segment. The dashed lines represent curves where $g$ and $U$ are held fixed and only the temperature is varied. These curves originate at the ordered fixed point $(\infty, 0)$ at $T=0$ and end at the disordered and unconstrained entropic fixed point $(0, \infty)$ at $T=\infty$. In the limit of $g / U \ll 1$, these curves become infinitesimally close to the $g / T$ and $T / U$ semiaxes, and the classification discussed in this paper applies. The universal physics occurs in regime (3.2c); therefore, in the region $T / U \ll 1, g / T \ll 1$ close to the origin of the coordinate system. The shaded region represents the scaling entropic region appearing in a constrained system due to the proximity to the constrained entropic critical point at the origin. (Top) This diagram encompasses scenarios I, III, and IV depending on the behavior of the system along the segment $0 \leqslant g / T \leqslant(g / T)_{\mathrm{c}}$ with $T / U=0$. (Bottom) Phase diagram corresponding to scenario II, where $(g / T)_{\mathrm{c}}=0$.

along the dashed line with $g / U \ll 1$, the correlation length first increases as one approaches the fixed point from the horizontal direction and only starts to decrease as one moves away along the vertical direction. The crossover temperature scale is set by Eq. (4.2), and it goes to infinity as $U \rightarrow \infty$.

\section{CONSTRAINED QUANTUM $X X Z$ HEISENBERG AND TRANSVERSE-FIELD ISING MODELS}

We are going to illustrate how the high-temperature critical scaling picture is realized for the constrained quantum $X X Z$ Heisenberg and transverse-field Ising models on the honeycomb lattice. The effective models are identical for both systems, the only difference lying with the order $n$ in $\Gamma / U$ needed to generate the effective "ring exchange" $\hat{H}_{\Gamma / U}^{(n)}$ in Eq. (2.7). So it suffices to analyze the constrained transverse-field Ising model (2.4) whose effective Hamiltonian (2.7) restricted to the subspace $\mathcal{H}_{0, U}$ takes the form

$$
\begin{aligned}
\hat{H}_{\mathrm{eff}}:= & -h \sum_{i=1}^{N} \hat{\sigma}_{i}^{z}-h_{s} \sum_{i=1}^{N}(-1)^{i} \hat{\sigma}_{i}^{z}-J \sum_{\langle i j\rangle} \hat{\sigma}_{i}^{z} \hat{\sigma}_{j}^{z} \\
& -\Gamma_{\mathrm{eff}}\left\{\sum_{\triangleright} \prod_{v=1}^{6} \hat{\sigma}_{i_{v}}^{x}+\cdots\right\},
\end{aligned}
$$

where the indices $i_{v}, v=1, \ldots, 6$, label the sites around an elementary hexagon $\square$. The coupling $h$ describes a uniform magnetic field, $h_{s}$ describes a staggered magnetic field, and $J$ describes an exchange interaction between nearest-neighbor sites of the honeycomb lattice. $J>0$ favors ferromagnetic $(\mathrm{F})$ order while $J<0$ favors antiferromagnetic (AF) order. Both the classical antiferromagnetic and ferromagnetic ground states are compatible with the constraint that the magnetization of each hexagonal plaquette has to be \pm 6 or 0 . These couplings $h, h_{s}$, and $J$ are three $g$-like couplings that we analyze below.

The temperature in the regime $(3.2 \mathrm{c})$ is small compared to $U$ and large compared to $\left|\Gamma_{\text {eff }}\right| \propto\left|\Gamma^{6} / U^{5}\right|$. The approximation of neglecting either violations of the constraint or the offdiagonal part of the quantum Hamiltonian should thus be a good starting point. If so, in the scaling regime $|h| / T,\left|h_{s}\right| / T,|g| / T, T / U \rightarrow 0$, the model reduces to a noninteracting Ising model on the honeycomb lattice, with the constraint that the magnetization of each hexagonal plaquette has to be \pm 6 or 0 . This model maps onto the noninteracting three-coloring model which was studied by Baxter and whose entropy can be computed exactly in the thermodynamic limit. ${ }^{14} \mathrm{He}$ also showed that the model exhibits algebraically decaying spatial correlations and as such is critical. We shall call this constrained entropic scaling limit $|h| / T,\left|h_{s}\right| / T,|g| / T, T / U \rightarrow 0$ the Baxter critical point. The long-wavelength, low-energy limit of this model is captured by a conformally invariant field theory with central charge $c=2 .^{16}$

As the temperature is lowered, the effects of the couplings $J, h, h_{s}$, and any other couplings compatible with the symmetries of Hamiltonian (5.1) need to be taken into account. That is, we need to decide what perturbations are relevant, marginal, and irrelevant at the Baxter critical point. Infinitesimally close to it in parameter space one can use perturbative renormalization group arguments. At a finite distance away these methods fail and one must rely on numerical tools to explore the stability of the Baxter critical point.

Natural choices for perturbations of the Baxter critical point are a uniform magnetic field $h$, a staggered field $h_{s}$, and 
a nearest-neighbor interaction $J$. The system is still exactly solvable in the presence of the $h_{s}$ coupling alone. In the presence of either the $h$ or $J$ coupling, the system is no longer exactly solvable, and we chose to resort to numerical transfer matrix calculations in order to investigate the fate of the Baxter critical point.

Since we are interested in distinguishing between criticality and any long-range-ordered or disordered gapped phase, a convenient choice is to measure the central charge of the system. This can be obtained from the coefficient of the largest finite-size scaling correction to the free energy of a semiinfinite system with periodic boundary conditions in the finite direction. ${ }^{17,18}$ The central charge is known to be strictly nonzero if the theory describing the long-wavelength behavior of the system is critical (massless). Numerically, a massive phase is signaled by the vanishing of the measured $c$, for the finite-size corrections vanish faster than in a critical system. (There are conformally invariant topological field theories with $c=0$, but the numerically measured $c=0$ is here more trivially an indication of a massive phase.) From similar calculations on the semi-infinite system one can also obtain the scaling dimensions of the operators in the conformal field theory describing the long-wavelength behavior of the system. In addition to providing a better understanding of the critical regime, the scaling dimensions are known to either vanish or diverge as the system becomes massive and they can be used to confirm the central charge results.

In order to compute the central charge and scaling dimensions of the system in a cylindrical geometry, we made use of transfer matrix techniques in combination with sparse matrix diagonalization routines from the free package ARPACK. Our results for the central charge are obtained either as a function of $h / T$ or as a function of $J / T$. We did not consider the case of $h$ and $J$ simultaneously present or the case when either is present together with $h_{s}$.

\section{A. Uniform field $h$ : Scenario I}

The numerical results for a uniform magnetic field $h$ are presented in Fig. 3 (as the transformation $h \rightarrow-h$ and $\hat{\sigma}_{i}^{z} \rightarrow-\hat{\sigma}_{i}^{z}$ leaves the Hamiltonian unchanged, it is sufficient to consider the case $h / T \geqslant 0)$. The dependence on $h / T$ of the central charge of a honeycomb lattice wrapped around a cylinder is shown in Fig. 3 (top), for different values of the cylinder radius. The dependence on $h / T$ of the two smallest scaling dimensions is shown in Fig. 3 (bottom).

The numerical calculations proceed in two steps, to be repeated for each value of the reduced magnetic field $h / T{ }^{17,18}$ First, the three largest eigenvalues $\Lambda_{j}^{(0)} \geqslant \Lambda_{j}^{(1)}$ $\geqslant \Lambda_{j}^{(2)}$ of the transfer matrix corresponding to the diameters $L_{j}=2 j, j=2, \ldots, 7$, of the cylinder are computed. Here $L_{j} / 2$ corresponds to the number of hexagonal plaquettes or, equivalently, $2 L_{j}$ is the number of spins in a row of the infinite cylinder. The largest eigenvalue $\Lambda_{j}^{(0)}$ yields the dimensionless free energy per spin,

$$
f_{j}=-\frac{1}{2 L_{j}} \ln \left(\Lambda_{j}^{(0)}\right),
$$

while the next two subleading eigenvalues $\Lambda_{j}^{(1,2)}$ yield the dimensionless excitation energies
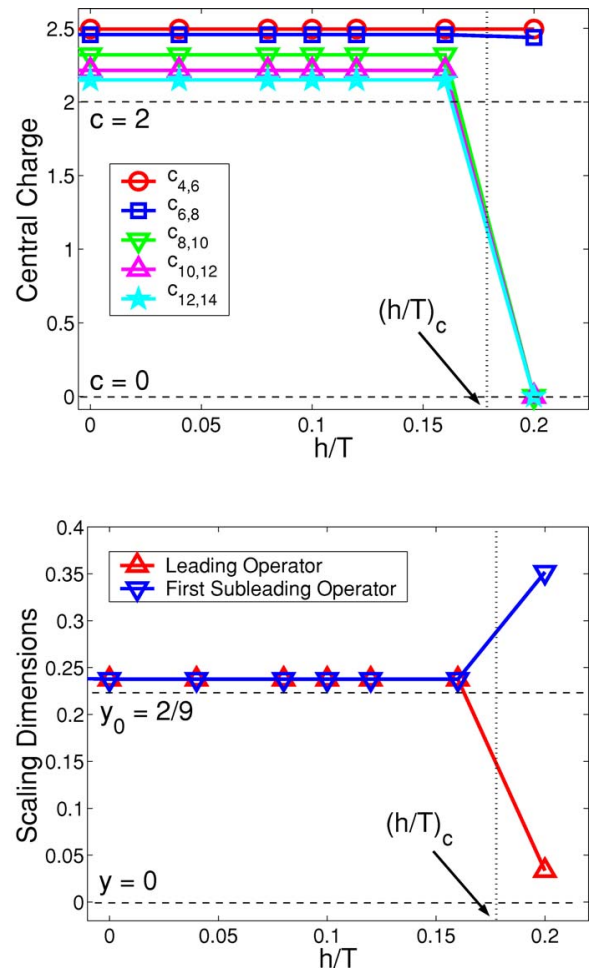

FIG. 3. (Color online) (Top) Behavior of the (finite-size) central charge $c_{L_{i}, L_{i+1}}$ as a function of $h / T$, obtained from finite-size scaling of the free energy computed via transfer matrix. From top to bottom, the different curves correspond to increasing diameters $L_{j}=4, \ldots, 14$ for the systems used in the scaling fit to compute the value of the central charge $c_{L_{i}, L_{i+1}}$. The system in presence of a field is symmetric upon the sign change $h \leftrightarrow-h$; therefore, only the $h>0$ axis is shown. Notice that the high-temperature criticality is robust with respect to a uniform field and it survives at large but finite temperatures. (Bottom) Behavior of the two smallest scaling dimensions allowed by the conformal field theory as a function of $h / T$. The extrapolated values for $L \rightarrow \infty$ are shown here for simplicity. The fact that both (degenerate) scaling dimensions remain constant in the critical regime suggests that the whole critical phase is described by the same conformal field theory. The lines between data points are guides to the eyes.

$$
\Delta f_{j}^{(k)}=\frac{1}{2 L_{j}} \ln \left(\frac{\Lambda_{j}^{(0)}}{\Lambda_{j}^{(k)}}\right), \quad k=1,2 .
$$

Second, a value of the (finite-size) central charge $c_{L_{i}, L_{i+1}}$ is obtained from the finite-size scaling fit performed on two consecutive values of the free energy-i.e., on data points of the type $\left\{\left(L_{j}, f_{j}\right), j=i, i+1\right\}$. This is repeated for $i=2$ to $i=6$. The (finite-size) scaling dimensions $y_{L_{i}, L_{i+1}}^{(k)}, k=1,2$, follow from finite-size scaling fits on two consecutive values of $\Delta f_{j}^{(k)}$-i.e., on data points of the type $\left\{\left(L_{j}, \Delta f_{j}^{(k)}\right), j=i, i+1\right\}$.

What we are after is not so much the finite-size values $c_{L_{i}, L_{i+1}}$ and $y_{L_{i}, L_{i+1}}^{(k)}$ as their values in the thermodynamic limit $L_{i} \rightarrow \infty(i \rightarrow \infty)$. We present finite-size data $c_{L_{i} L_{i+1}}$ in Fig. 3 (top) to illustrate the finite-size corrections while we present the extrapolated values $y^{(k)} \equiv \lim _{i \rightarrow \infty} y_{L_{i}}^{(k)} L_{i+1}$ in Fig. 3 (bottom). The dependence on $h / T$ of the central charge 
$c \equiv \lim _{i \rightarrow \infty} c_{L_{i}, L_{i+1}}$ that we deduce from Fig. 3 (top) is

$$
c= \begin{cases}2, & 0 \leqslant \frac{h}{T}<\left(\frac{h}{T}\right)_{\mathrm{c}}, \\ 0, & \left(\frac{h}{T}\right)_{\mathrm{c}}<\frac{h}{T} .\end{cases}
$$

From Fig. 3 one reads that the Baxter critical point is robust to the introduction of a uniform magnetic field. According to Fig. 3 (top), the system remains critical with central charge $c=2$ over the finite interval $0 \leqslant h / T \leqslant(h / T)_{c}$, before entering an ordered phase through a (first-order) phase transition at $(h / T)_{\mathrm{c}}$. This is confirmed by the behavior of the scaling dimensions shown in Fig. 3 (bottom), which seem to rapidly vanish or diverge across the transition at $(h / T)_{\mathrm{c}}$, respectively. Also, according to Fig. 3 (bottom), the smallest scaling dimensions are unchanged along the segment $0 \leqslant h / T \leqslant(h / T)_{\text {c }}$. These numerical results support the conclusion that a small coupling $h / T$ is irrelevant at the Baxter critical point and scenario I is realized along the segment.

This behavior is perhaps surprising if compared to the effect of a uniform magnetic field in the unconstrained Ising model on the honeycomb lattice. In that case, the uniform magnetic field is a relevant perturbation that causes the system to order at any finite temperature. The origin of this difference is due to the large depletion of configurations with finite magnetization induced by the projective action of the constraint. The entropy of the system as a function of magnetization $m$ seems to acquire a cusp at $m=0$ that leads to a strong first-order transition at finite temperature in the presence of a uniform magnetic field.

\section{B. Staggered field $h_{s}$ : Scenario II}

The case of the staggered magnetic field $h_{s}$ has been solved exactly by Baxter ${ }^{14}$ in the limit $T / U \rightarrow 0, h_{s} / T$ arbitrary. The model exhibits an infinite-order phase transition as $h_{s} / T \rightarrow 0$. The staggered field is a marginally relevant coupling to the Baxter critical point $h_{s} / T, h_{s} / U, T / U \rightarrow 0$ (Ref. 16); i.e., a staggered magnetic field realizes scenario II of Sec. IV C. In particular, this implies that any small staggered field $h_{s} / T$ induces an exponentially large correlation length

$$
\xi_{\text {II }}\left(h_{s} / T\right) \sim \mathfrak{a} \exp \left(\alpha_{h_{s}} \frac{T}{h_{s}}\right),
$$

where $\alpha_{h_{s}}$ is some dimensionless number.

The divergence of the correlation length (5.4) as $h_{s} / T \rightarrow 0$ is cut off at the crossover temperature

$$
T_{\text {cross }} \sim \sqrt{U h_{s}} \text {. }
$$

This estimate follows from the finite correlation length induced by the fact that $T / U$, although large, is finite. Indeed, the Baxter critical point is unstable to constraint-violating defects that appear as soon as $T / U$ is finite. The dependence on $T / U$ of the corresponding correlation length $\xi_{\mathrm{ce}}(T / U)$ is governed by the most relevant operator that introduces violations of the constraint in the spin language. This operator
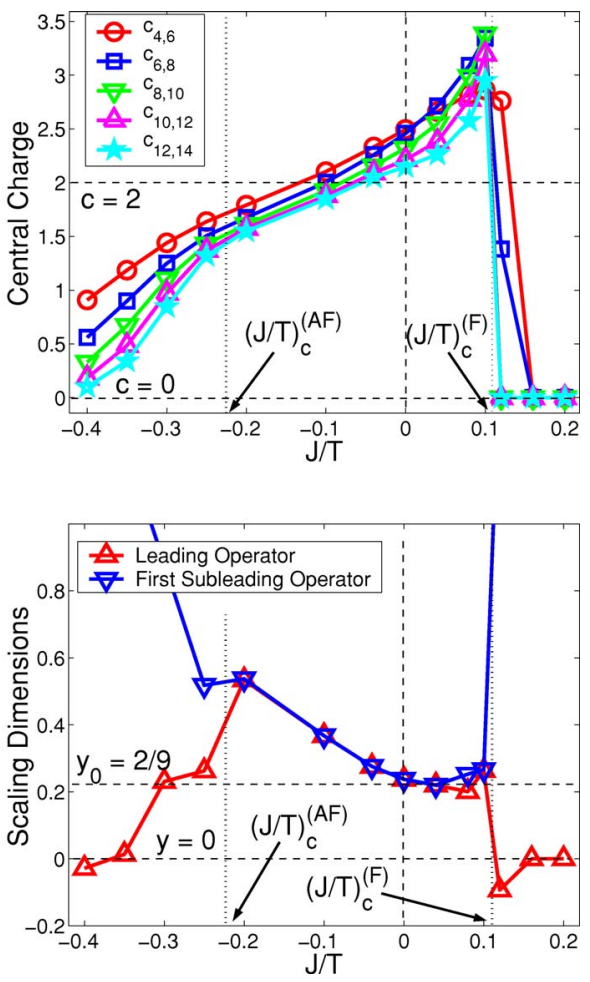

FIG. 4. (Color online) (Top) Behavior of the (finite-size) central charge $c_{L_{i}, L_{i+1}}$ as a function of $J / T$, obtained from finite-size scaling of the free energy computed via transfer matrix. From top to bottom, the different curves correspond to increasing diameters $L_{j}=4, \ldots, 14$ for the systems used in the scaling fit to compute the value of the central charge $c_{L_{i}, L_{i+1}}$. Positive values of $J$ correspond to a ferromagnetic (F) coupling, while negative values correspond to an antiferromagnetic (AF) coupling. Notice that the hightemperature criticality is robust with respect to uniform nearestneighbor interactions and it survives at large but finite temperatures both for positive and negative $J$. Antiferromagnetic interactions, however, deeply affect the critical behavior, inducing what appears to be a continuously varying central charge. (Bottom) Behavior of the two smallest scaling dimensions allowed by the conformal field theory as a function of $J / T$. The extrapolated values for $L \rightarrow \infty$ are shown here for simplicity. Contrary to the uniform field case, the scaling dimensions vary on the AF side $(J / T)_{\mathrm{c}}^{(A F)} \leqslant J / T \leqslant 0$. Variations of the scaling dimensions on the $\mathrm{F}$ side $0 \leqslant J / T<(J / T)_{\mathrm{c}}^{(F)}$ cannot be resolved numerically. The lines between data points are guides to the eyes.

corresponds to the insertion of fractional vortices in the continuum theory that describes the Baxter critical point. The scaling dimension $y_{U}$ of this operator is given by $y_{U}=2 / 9$ according to Ref. 19 in the case when there are no interactions added to the model. This is indeed in agreement with our numerical results for $h / T, J / T \rightarrow 0$ (see Figs. 3 and 4). The estimate (4.1a) thus becomes

$$
\xi_{\mathrm{ce}}(T / U) \sim \mathfrak{a} \exp \left(\alpha_{U} \frac{9 U}{16 T}\right)
$$

and the crossover temperature (5.5) follows from solving Eq. (4.2) with the help of Eqs. (5.4) and (5.6). 


\section{Nearest-neighbor interaction $\boldsymbol{J}>\mathbf{0}$ : Scenario III}

The numerical results for a uniform nearest-neighbor interaction $J$ between the Ising spins are presented in Fig. 4. The dependence on $J / T$ of the central charge of a honeycomb lattice wrapped around a cylinder is shown in Fig. 4 (top), for different values of the cylinder radius. The dependence on $J / T$ of the two smallest scaling dimensions is shown in Fig. 4 (bottom).

We consider first the ferromagnetic side of the interaction, $J>0$. According to Fig. 4 (top), for small but finite $J / T$, the central charge remains larger than or equal to the noninteracting value $c=2$, until the central charge drops to zero abruptly. This suggests that the correlation length remains infinite over the finite interval $0 \leqslant J / T<(J / T)_{\mathrm{c}}^{(F)}$, until the system undergoes a strong first-order transition at $(J / T)_{\mathrm{c}}^{(F)}$. This is confirmed by the behavior of the scaling dimensions, which seem to rapidly vanish or diverge across the transition at $(J / T)_{\mathrm{c}}^{(F)}$, respectively. Moreover, Fig. 4 (bottom) suggests that the lowest scaling dimensions vary continuously with $J / T$, even though numerics alone cannot be deemed conclusive on this issue.

The behavior of the central charge in the critical range $0 \leqslant J / T<(J / T)_{\mathrm{c}}^{(F)}$ is different from the uniform field case, as $c_{L_{i}, L_{i+1}}$ is seen to grow significantly upon approaching the critical value $(J / T)_{\mathrm{c}}^{(F)}$ for a fixed system size $L_{i}$. This growth can be explained by exponential corrections to finite-size scaling that are known to occur at a first-order phase transition. Indeed, extrapolating the curves in Fig. 4 in the limit $L_{j} \rightarrow \infty$ yields an approximately constant value of $c=2$ over the interval $0 \leqslant J / T<(J / T)_{\mathrm{c}}^{(F)}$. The extrapolated curve is, however, rather noisy due to the limited range of numerically accessible system sizes and it is not shown here. The reason why these corrections are so strong in the case of a nearestneighbor ferromagnetic perturbation compared to the case of a uniform magnetic field deserves further study.

Our interpretation of Fig. 4 is that, in the thermodynamic limit, the central charge is constant on the segment $0 \leqslant J / T<(J / T)_{\mathrm{c}}^{(F)}$ while the scaling dimensions are not. If so, the segment $0 \leqslant J / T<(J / T)_{\mathrm{c}}^{(F)}$ realizes a line of critical points; i.e., $J / T$ is a marginal interaction along this segment, thus realizing scenario III. This interpretation agrees with the perturbative RG calculation from Ref. 20.

The equilibration properties of the constrained $X X Z$ Heisenberg or transverse-field Ising model in the ferromagnetically ordered phase exhibit rather peculiar features and have been discussed in Ref. 9. Based on those results, we expect quantum glassiness to appear in the system when the temperature is lowered across the transition to the ferromagnetically ordered phase, at least as long as the transition temperature is small enough for the $U$-violating defects not to play a significant role in the equilibration process (Fig. 5).

\section{Nearest-neighbor interaction $\boldsymbol{J}<0$ : Scenario IV}

The behavior of the central charge is even more surprising on the antiferromagnetic side of the interaction $J<0$. The values taken by $c_{L_{i}, L_{i+1}}$ in Fig. 4 (top) are seen to drop below $c=2$ for $J / T<-0.1$ as soon as the system size is sufficiently

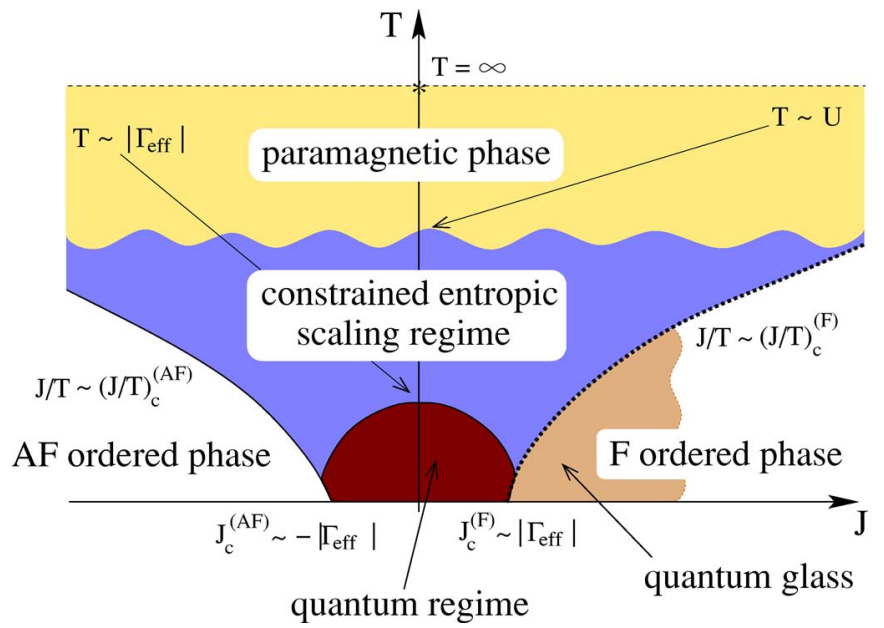

FIG. 5. (Color online) Illustration of the phase diagram of the constrained Ising model in the presence of a nearest-neighbor coupling $J$, discussed in Sec. V. $U$ is held fixed in the figure, and only two out of the three parameters of the model—namely, $J$ (horizontal axis) and $T$ (vertical axis) - are shown. The effects of the quantum energy scale $\Gamma$ become dominant only at very small values of the coupling $J$, in the interval $-\left|\Gamma_{\text {eff }}\right| \lesssim J \lesssim\left|\Gamma_{\text {eff }}\right|$, and for very small temperatures $T \lesssim\left|\Gamma_{\text {eff }}\right|$, giving rise to the quantum regime shown in the picture. At low temperatures, but for $|J| \gg\left|\Gamma_{\text {eff }}\right|$, the system exhibits two classical ordered phases, a ferromagnetic $(\mathrm{F})$ one for $J>0$ and an antiferromagnetic (AF) one for $J<0$. While the transition to the AF ordered phase is continuous, the transition to the $\mathrm{F}$ ordered phase is strongly first order (dotted line). As discussed in Ref. 9, quantum glassiness is expected to appear in the system when the temperature is lowered across the transition to the F-ordered phase, at least as long as the transition temperature is small enough for the $U$-violating defects not to play a significant role in the equilibration process.

large. As the diameter of the cylinder is increased from $L_{i}=4$ to $L_{i}=14$, the dependence of $c_{L_{i}, L_{i+1}}$ on $J / T$ seems to indicate the existence of two distinct regimes for $J / T$. On the one hand, the $c_{L_{i}, L_{i+1}}$ appear to collapse onto a nonvanishing value of $c$ for sufficiently large $J / T$ (close to $J / T=0$ ) in the thermodynamic limit. On the other hand, consecutive $c_{L_{i}, L_{i+1}}$ remain well separated from each other for sufficiently small (negative) $J / T$, thereby suggesting a vanishing limiting value $c$ for the central charge in the thermodynamic limit.

We interpret our finite-size simulations as signaling the existence of a (continuous) transition at a finite $(J / T)_{\mathrm{c}}^{(A F)} \simeq-0.2$, which is in agreement with variational mean-field results by Cirillo et al. in Ref. 21. Our simulations for the two smallest scaling dimensions also agree with this interpretation. While they are degenerate and they vary continuously in the temperature region $(J / T)_{\mathrm{c}}^{A F}<J / T<0$, they split and rapidly vanish or diverge as soon as $J / T<(J / T)_{\mathrm{c}}^{(A F)}$, respectively. As for our numerical results for the central charge, we interpret them as indicative of one of three different possibilities. The first possibility is that the phase transition at $(J / T)_{\mathrm{c}}^{(A F)}$ separates a phase with vanishing central charge below $(J / T)_{\mathrm{c}}^{A F}$ and a line of critical points with continuously varying central charge above $(J / T)_{c}^{(A F)}$ that interpolate between the values $c=3 / 2$ at $(J / T)_{\mathrm{c}}^{(A F)}$ and $c=2$ at 
infinite temperature. The second possibility is that the finitesize data when $(J / T)_{\mathrm{c}}^{(A F)}<J / T<0$ are the signature of an infinite sequence of stepwise increases of the central charge in the thermodynamic limit that interpolates between the values $c=3 / 2$ at $(J / T)_{\mathrm{c}}^{(A F)}$ and $c=2$ at infinite temperature. (See Ref. 22 for a possibly related phenomenon.) And finally, the third possibility is that the finite-size data for $c_{L_{i}, L_{i+1}}$ collapse in the thermodynamic limit to the central charge $c=0$ when $J / T<(J / T)_{\mathrm{c}}^{(A F)}$ and $c=3 / 2$ when $(J / T)_{\mathrm{c}}^{(A F)}<J / T<0$. The plateau with $c=3 / 2$ would correspond to a conformal field theory built out of three Majorana fermions and endowed with a supersymmetry. More accurate simulations-i.e., simulations that can access larger diameters of the cylinder on which the honeycomb lattice is wrapped-are required to select which of these possibilities corresponds to the correct thermodynamic limit.

The phase diagram for the constrained Ising model in the presence of a nearest-neighbor interaction is summarized in Fig. 5.

\section{E. Possible experimental realization of the constrained Ising model in a transverse field}

A possible physical realization of the constrained classical Ising model has been discussed in Refs. 19 and 20 in the form of lattices of superconducting devices with broken time-reversal symmetry. Finding an experimental probe mimicking a staggered magnetic field might allow the observation of a correlation length that increases with temperature over a large window of temperatures in the regime (3.2c).

\section{SQUARE-LATTICE DIMER MODEL}

We are now going to illustrate how the high-temperature critical scaling picture is realized for the bosonic model in the example (2.5). The effective Hamiltonian (2.7) restricted to the subspace $\mathcal{H}_{0, U}$ takes the form ${ }^{23}$

$$
\begin{aligned}
\hat{H}_{\mathrm{eff}}:= & v \sum_{i=1}^{N}\left(\hat{n}_{i, i+x} \hat{n}_{i+y, i+y+x}+\boldsymbol{x} \leftrightarrow \boldsymbol{y}\right) \\
& -\Gamma_{\mathrm{eff}} \sum_{i=1}^{N}\left[e^{+i\left(\hat{a}_{i, i+x}+\hat{a}_{i+y, i+y+x}\right)-i(x \leftrightarrow y)}+\text { H.c. }\right]+\cdots .
\end{aligned}
$$

Here, $\hat{a}_{i, i+e}$ is the Hermitian operator canonically conjugate to the local bosonic number operator $\hat{n}_{i, i+e}$; i.e., their commutator is the $\mathrm{C}$ number $\mathrm{i}$. Under the assumption that $|v| \sim|t| \ll U$ the coupling $\Gamma_{\text {eff }} \propto t^{2} / U$ of the off-diagonal term in the effective Hamiltonian (6.1) is much smaller than $v$. If we relax this assumption by allowing for all possible values of the ratio $v / \Gamma_{\text {eff }}$, Eq. (6.1) is nothing but the square-lattice quantum dimer model introduced by Rokhsar and Kivelson in Ref. 24.

We are after the high-temperature universal behavior of the regime (3.2c). To this purpose, we consider first the constrained entropic scaling limit $|v| / T, T / U \rightarrow 0$ for which the model reduces to the square-lattice classical noninteracting dimer model, which was studied by Kasteleyn in Ref. 15. He showed that the entropy can be computed exactly in the thermodynamic limit. He also showed that the model exhibits algebraically decaying spatial correlations, and, as such, it is critical. We shall call the constrained entropic scaling limit $|v| / T, T / U \rightarrow 0$ the Kasteleyn critical point.

The Kasteleyn critical point is captured by a height model which, in the long-wavelength limit, is described by the twodimensional conformally invariant field theory ${ }^{23}$

$$
S=\pi K \int d^{2} \boldsymbol{x}|\nabla \phi|^{2},
$$

with stiffness specified by

$$
K=\frac{1}{2}
$$

and central charge

$$
c=1 .
$$

A microscopic dimer is represented in the field theory (6.2) by a linear combination of two field operators. The first one is the charge $q_{e}= \pm 1$ "vertex operator" $\exp \left(\mathrm{i} 2 \pi q_{e} \phi\right)$ where the sign assignment has to do with the lattice being bipartite. The second one is the "dipolar operator" $\boldsymbol{e} \cdot \nabla \phi$ where $\boldsymbol{e}$ is one of the two basis vectors of the square lattice such that $(i, i+\boldsymbol{e})$ is the pair of sites covered by the dimer (for some site $i$ ). The two-point correlation function

$$
\left\langle e^{\mathrm{i} 2 \pi(\phi(x)-\phi(\boldsymbol{y}))}\right\rangle_{K} \sim\left(\frac{\mathfrak{a}}{|\boldsymbol{x}-\boldsymbol{y}|}\right)^{1 / K}
$$

decays with the exponent 2 when $K=1 / 2$. The two-point correlation function

$$
\langle(\boldsymbol{e} \cdot \boldsymbol{\nabla} \phi(\boldsymbol{x}))(\boldsymbol{e} \cdot \boldsymbol{\nabla} \phi(\boldsymbol{y}))\rangle_{K} \sim\left(\frac{\mathfrak{a}}{|\boldsymbol{x}-\boldsymbol{y}|}\right)^{2}
$$

decays with the exponent 2 for any stiffness $K>0$. The value (6.2b) is thus special in that the scaling dimensions of the charge $q_{e}= \pm 1$ vertex and dipolar operators are degenerate and equal to 1 .

A monomer at site $i$ of the square lattice is a defect in the dimer covering since site $i$ is not the end point of a dimer. A finite concentration of monomers is represented in the field theory (6.2) by the local charge $q_{m}= \pm 1$ vertex operator $\exp \left(\mathrm{i} 2 \pi K q_{m} \varphi\right)$ for the field $\varphi$ dual to $\phi$,

$$
\left(\frac{\partial \varphi}{\partial x}, \frac{\partial \varphi}{\partial y}\right)=\left(\frac{\partial \phi}{\partial y},-\frac{\partial \phi}{\partial x}\right) .
$$

The two-point correlation function

$$
\left\langle e^{\mathrm{i} 2 \pi K[\varphi(\boldsymbol{x})-\varphi(\boldsymbol{y})]}\right\rangle_{K} \sim\left(\frac{\mathfrak{a}}{|\boldsymbol{x}-\boldsymbol{y}|}\right)^{K}
$$

decays with the exponent $1 / 2$ when $K=1 / 2$. The charge \pm 1 monomer is represented by a strongly relevant operator with scaling dimension $1 / 4$. 


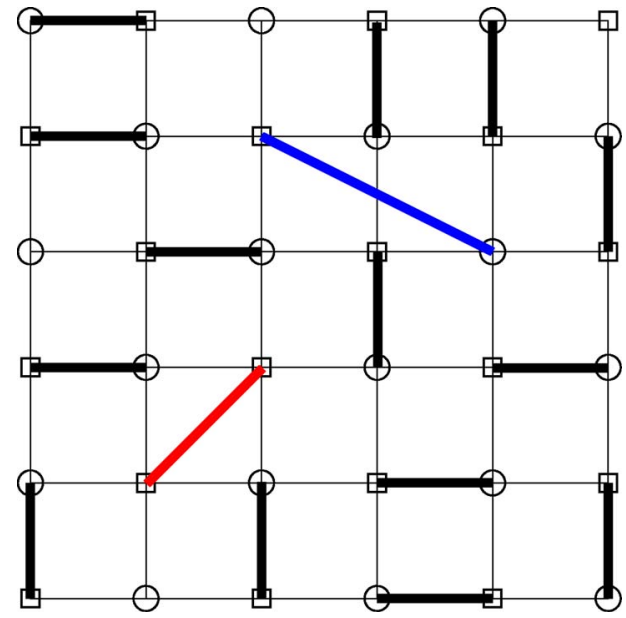

FIG. 6. (Color online) Example of longer dimers that can be thought of as pairs of monomers chained together. When the end points of a long dimer sit at sites within the same sublattice, the chained monomers have equal charges. When the end points sit at opposite sublattices, the chained monomers have opposite charges.

In the following we will focus on the temperature region where the quantum effects are negligible for the thermodynamic properties of the system-i.e., when $\left|\Gamma_{\text {eff }}\right| \ll T \ll U$.

\section{A. Case $v=0$ in bipartite lattices: Scenarios II and III}

It is useful to first consider the situation when $v=0$ and add some other perturbations to dimer models like those recently studied by Sandvik and Moessner in Ref. 25. We introduce a small fugacity for dimers to cover bonds that, although of finite length, extend beyond nearest-neighbor bonds. Consider separately two cases: (1) when the long bonds break the bipartiteness of the lattice by connecting sites in the same sublattices and (2) when the bonds preserve the bipartite nature of the lattice.

We interpret these perturbations within the framework of the field theory as follows. The introduction of appropriate chemical potentials leads to a small fraction of the longer dimers. If one of these longer dimers is simply removed from a configuration, one is left with two monomers in the system. So a longer dimer can be thought of as two monomers at its end points that are chained together (see Fig. 6). In case (1) a longer dimer connecting sites in the same sublattice can be interpreted as a pair of chained monomers with equal charges. The operator product expansion (OPE) of the corresponding charge $q_{m}= \pm 1$ vertex operators leads to charge $q_{m}= \pm 2$ vertex operators. These composite operators have scaling dimension $2^{2} \times K / 2=1$ when $K=1 / 2$. Thus, they are relevant and they open a gap in the system. The underlying structure consists of a disordered arrangement of dimers with short range correlations. Hence, the Kasteleyn critical point should be strongly unstable to perturbations that break the sublattice symmetry. ${ }^{26}$ The simplest perturbation of this type is to allow next-nearest-neighbor bonds to be covered by dimers. Other analytical arguments and numerics are consistent with this expectation. ${ }^{25,26}$ Therefore, scenario II is realized upon the introduction of any finite chemical potential for second-neighbor dimers in the system.
In case (2) a longer dimer connecting sites in opposite sublattices can be interpreted as a pair of chained monomers with opposite charges. The OPE of the corresponding vertex operators (when all the possible directions of the long dimer are added) will lead to a $|\nabla \phi|^{2}$ term that renormalizes the stiffness. This is also consistent with the numerical results of Refs. 25 and 26. Scenario III is thus realized.

We would like to note that violations of the constraint in the form of monomers are relevant perturbations that drive the system away from the the Kasteleyn critical point, as in all the situations discussed is Sec. IV. The finite correlation length (4.1a) thereby generated is given by

$$
\xi_{\mathrm{ce}}(T / U) \sim \mathfrak{a} \exp \left(\alpha_{U} \frac{4 U}{7 T}\right)
$$

when $T \ll U$.

\section{B. Case $v \neq 0$ in bipartite lattices: Scenario III}

We are going to argue on the basis of numerics that scenario III can also be realized by perturbing the Kasteleyn critical point with the interaction $v$ in Eq. (6.1).

To this end, observe that $\hat{H}_{v}$, defined by Eq. (2.5a), counts the total number of elementary square plaquettes of the square lattice that are flippable in the dimer basis representation. Here, a flippable plaquette is an elementary square plaquette that has two occupied edges (dimers). This model has already been studied by Alet $e t$ al. in Ref. 27 for negative values of the coupling constant $v$. Here we consider both positive and negative values, and good agreement with the previous results is found where they overlap. ${ }^{27}$

As exact analytical results are no longer available, we use a numerical approach similar to the one described in Sec. V. We compute the central charge of the system as well as the scaling dimensions of two specific operators via transfer matrix techniques, using finite size scaling fits. The accessible system sizes are $L_{j}=2 j, j=3, \ldots, 8$, where $L_{j}$ is the number of square plaquettes across the periodic direction of the system or, equivalently, $2 L_{j}$ is the number of edges. As discussed by Alet et al. in Ref. 27, the critical regime of this system is captured by a $c=1$ two-dimensional conformal field theory of the Coulomb gas type, with continuously varying stiffness. They also computed the scaling dimensions of some known operators in the conformal field theory; namely, those corresponding to the electric and magnetic vortices in the Coulomb gas picture. From these measurements one can then obtain the values of all other scaling dimensions present in the conformal field theory and discuss the nature of the critical phase and of the phase transitions.

The central charge as a function of the coupling constant $v / T$ is plotted in Fig. 7 (top). Thanks to the good convergence in the $L \rightarrow \infty$ limit, we report the extrapolated values of $c$ instead of each separate curve for increasing system size. From the existence of a $c=1$ plateau

$$
(v / T)_{\mathrm{c}}^{\text {(columnar) }} \leqslant v / T \leqslant(v / T)_{\mathrm{c}}^{\text {(staggered) }}
$$

that extends to the left and right of the Kasteleyn fixed point $v / T, T / U \rightarrow 0$, we infer that criticality is preserved upon in- 
(a)

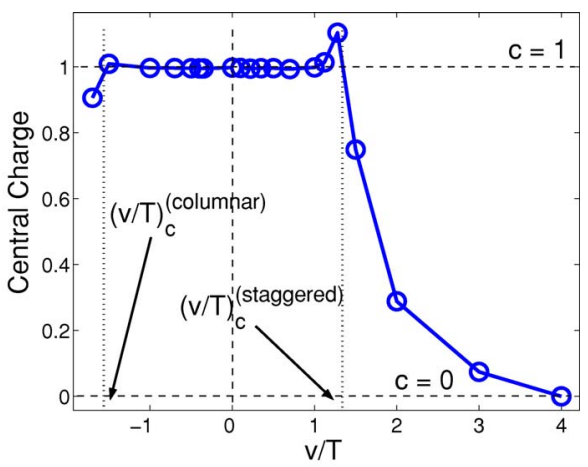

(b)

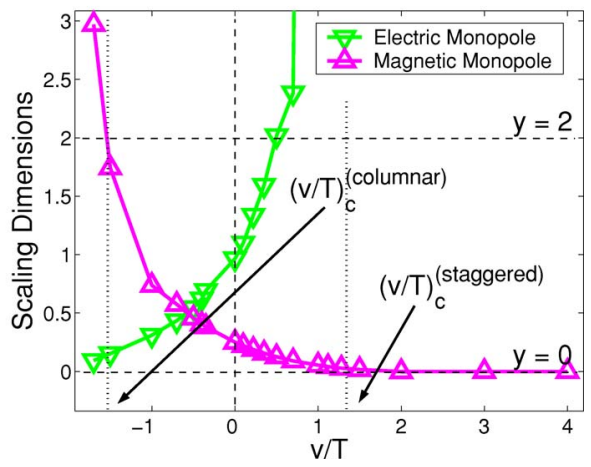

FIG. 7. (Color online) (Top) Behavior of the extrapolated central charge as a function of $v / T$, obtained from finite-size scaling of the free energy computed via transfer matrix. (Bottom) Behavior of the scaling dimensions corresponding to the electric and magnetic vertex operators as a function of $v / T$, also obtained from finite-size scaling arguments and transfer matrix calculations. Observe that the scaling dimensions of the electric and magnetic vertex operators are 1 and $1 / 4$, respectively, when $v / T=0$. The lines between data points are guides to the eyes.

troducing a small but finite coupling $v / T$. Moreover, the behavior of the scaling dimensions presented in Fig. 7 (bottom) tells us that the stiffness $K$ in the critical theory (6.2a) varies continuously along the line of critical points. Therefore we conclude that the introduction of the coupling $v$ realizes scenario III.

The transition at $T=T_{\mathrm{c}}^{\text {(columnar) }}$ has been characterized by Alet et al. The quasi-long-range-ordered phase (6.8) undergoes a Kosterliz-Thouless transition at $(v / T)_{\mathrm{c}}^{\text {(columnar) }}$ to an ordered phase. This phase is characterized by an alignment of parallel dimers along columns or rows and is therefore called the columnar phase.

On the other side, for $v>0$, our results show that the quasi-long-range-ordered phase (6.8) terminates at $T=T_{\text {c }}^{\text {(staggered) }}$ where it undergoes a first-order phase transition to an ordered phase. This phase is characterized by a staggering of parallel dimers along two consecutive columns or rows and is therefore called the staggered phase.

The phase diagram in the regime $(3.2 \mathrm{c})$ has the same topology as the one for the constrained Ising model in the presence of a nearest-neighbor interaction (Fig. 5).

\section{QUANTUM-DOMINATED REGIME $|g| \ll\left|\Gamma_{\text {eff }}\right|$}

In Secs. III-VI we assumed that $\left|\Gamma_{\text {eff }}\right|$ is the smallest energy scale in the problem. This is to be expected whenever
$\Gamma_{\text {eff }}=\Gamma(\Gamma / U)^{n-1}$ is highly suppressed because of the order $n$ needed for virtual processes to return to an allowed configuration. Even if we initially had $|g|<|\Gamma|$, it is likely that $\left|\Gamma_{\text {eff }}\right| \ll|g|<|\Gamma|$, since $|\Gamma| \ll U$. However, if the coupling constant $|g|$ for those terms that commute with the constraint is small compared to $\left|\Gamma_{\text {eff }}\right|$ or, in particular, if $g=0$, the discussion above must be revisted.

Let us consider here the case $g=0$ for simplicity. Independently of whether $\Gamma_{\text {eff }}$ is suppressed with respect to $\Gamma$ or not, there are now only three regimes of temperatures:

$$
\begin{aligned}
& T \ll\left|\Gamma_{\text {eff }}\right| \ll U, \\
& \left|\Gamma_{\text {eff }}\right| \ll T \ll U, \\
& \left|\Gamma_{\text {eff }}\right| \ll U \ll T .
\end{aligned}
$$

The constraint, as well as all other energy scales in the system, becomes negligible in regime $(7.1 \mathrm{c})$ and the resulting physics is that of a featureless high-temperature phase-say, a paramagnetic phase if the degrees of freedom are exclusively magnetic.

As the temperature is lowered down to the regime (7.1b), the system is still classical (at least from the point of view of its thermodynamic properties) but the constraint is now enforced. The physics is controlled by the proximity to the constrained entropic critical point at $\left|\Gamma_{\text {eff }}\right| / T, T / U \rightarrow 0$.

Finally, in the regime (7.1a) the system is fully quantum mechanical.

When $|g|$ is finite but much smaller than $\left|\Gamma_{\text {eff }}\right|$, these arguments should still be valid. Therefore, we can draw a qualitative phase diagram for a generic strongly constrained quantum system at fixed $g$ as a function of $\Gamma_{\text {eff }}$. In Fig. 8 we represent the phase diagram of a system exhibiting a zerotemperature phase transition at a finite value of the ratio $\Gamma_{\text {eff }} / g$. Notice that the shaded dome around the origin or parameter space contains both the classical ordered phase that onsets when $|g|$ becomes larger than $\left|\Gamma_{\text {eff }}\right|$ and the quantum critical scaling regime that is expected to appear in a cone-shaped region above the zero-temperature quantumcritical point. The actual details of this shaded region are highly system specific.

It is worth mentioning that the existence of a zerotemperature phase transition at $\Gamma_{\text {eff }} \sim \pm|g|$ is by no means a necessity. It is also possible - for example - that the coupling $\Gamma_{\text {eff }}$ never destroys the ordered phase determined by the diagonal coupling $g$ at zero temperature, e.g., if it favors the same type of order. In this case there would be no quantumgapped phase in Fig. 8 and the dome above the origin of parameter space would stretch out from $(-\infty, 0)$ to $(\infty, 0)$, without any phase transition at $( \pm \infty, 0)$. The phase under the dome would be uniform and determined by the (fixed) value of $g$. This is so, for example, in the quantum Hamiltonian (6.1) for any fixed value of $g<0$, where the columnar ordered phase is stable at all values of $\Gamma_{\text {eff }}$ according to the numerical results in Ref. 28 . 


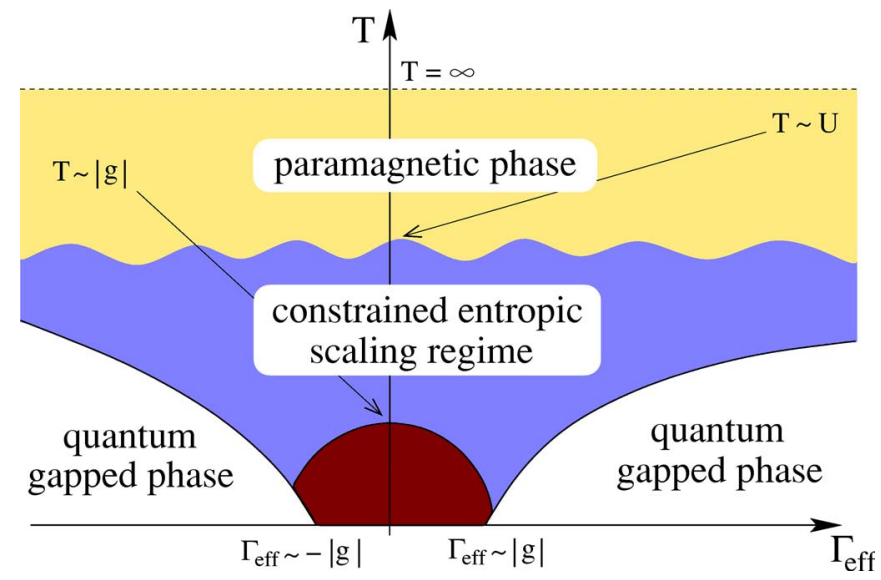

FIG. 8. (Color online) Generic phase diagram for a strongly constrained quantum system that satisfies conditions (1)-(6) from Sec. I. Parameter space encodes the competition between two energy scales, the temperature $T$, and a characteristic energy scale $\Gamma_{\text {eff }}$ given by the effective coupling of the off-diagonal term in the Hamiltonian, while $g$ and $U$ are held fixed. Here we chose to represent the case of a system exhibiting a zero-temperature phase transition at a finite value of the ratio $\Gamma_{\text {eff }} / g$. A quantum-critical scaling regime, if it exists, is restricted to a region represented by the upper half of a disk of radius $\Gamma_{\text {eff }}$ and centered at the origin $(g, T)=(0,0)$ of parameter space. As in the case of Fig. 1, the system exhibits a constrained entropic scaling regime at temperatures intermediate between the small characteristic quantum energy scale $\Gamma_{\text {eff }}$ and the large characteristic energy scale $U$ set by a strong constraint. At a fixed temperature, the constrained entropic scaling regime terminates in a phase transition that needs not be continuous upon increasing $\left|\Gamma_{\text {eff }}\right|$. At fixed $\Gamma_{\text {eff }}$, the constrained entropic scaling regime crosses over to the conventional high-temperature phasesay, a paramagnetic one for spin degrees of freedom-when $T$ is of the order of $U$.

\section{CONCLUSIONS}

In this paper we have presented a mechanism that leads to a large temperature regime where critical scaling behavior appears as a consequence of having a large energy scale $U$ in the problem. Hard constraints are imposed on the system in the limit $U \rightarrow \infty$ that project the original Hilbert space onto a space of allowed configurations satisfying the constraints. Critical behavior in the $U \rightarrow \infty$ limit occurs when correlations functions, calculated as uniform averages over all of these projected states, are algebraically decaying in space. We call the limit $U \rightarrow \infty$ followed by $T \rightarrow \infty$ at which criticality emerges a constrained entropic critical point. For large but finite $U$ and in the presence of other couplings $|g|,|\Gamma| \ll U$ in the problem, the physics at temperatures large compared to all other couplings but small compared to $U$ is still controlled by the proximity to the constrained entropic critical point. Indeed, this hierarchy among the couplings opens a rather large window of temperatures for which a constrained entropic critical regime exists as a consequence of the proximity to the constrained entropic critical point. We expect the constrained entropic critical regime to be qualitatively different in general from the quantum-critical regime associated with a putative quantum-critical point at zero temperature. The picture is summarized in Fig. 1 .
The mechanism for criticality at high temperature discussed here should be applicable to some, but not all, problems with a large dominant energy scale. Examples can be found in frustrated magnets-say, pyrochlore antiferromagnets where a magnetic field that induces a magnetization plateau at half the value of full magnetization supplies a strong constraint. ${ }^{29-31}$ Furthermore, since the order $n$ of a virtual process leading to the effective kinetic quantum term $\Gamma_{\text {eff }}=\Gamma(\Gamma / U)^{n-1}$ is usually large (it is of order 9 in Ref. 30), the window of temperatures for which the system is in the quantum regime can be extremely small $\left(T<\left|\Gamma_{\text {eff }}\right| \ll|\Gamma|\right)$. Hence, in practice, even for the smallest temperatures accessible experimentally, these constrained systems should either order or be within the constrained entropic critical regime. What determines whether the system orders or not is the presence of another energy scale $g$, set by the coupling strengths of additional terms in the Hamiltonian that commute with the $U$ term imposing the constraint.

Another example for which the idea of high-temperature criticality may apply is that of the fluctuations about a socalled $d$ density wave (DDW). ${ }^{32}$ In this instance, the order may form locally at some large energy $\Delta_{d}$, but the current loop directions may fluctuate. 6,33 At low temperatures, the system should order in a given current pattern. Above the global ordering temperature, this system should display highenergy constrained entropic criticality, because it resembles an ice model as long as $T$ is below $\Delta_{d}$.

In the single-band Hubbard model, with a strong local on-site repulsion only, there is no constrained entropic critical point in the $U \rightarrow \infty$ limit. It has been recently proposed by Phillips and co-workers that the order in which the limit $U \rightarrow \infty$ and the thermodynamic limit $L \rightarrow \infty$ ( $L$ being the linear size of the system) are taken has implications for hole transport in the single-band Hubbard model. ${ }^{34}$ The issue is that, for finite $U$, there is a characteristic length scale associated with the distance between doubly occupied sites, and hence for system sizes greater than this distance one has a finite density of such "defects." The appearance of this extra length scale was suggested as a way to resolve the issue of the breakdown of the one-parameter scaling picture for quantum criticality in the cuprates. ${ }^{35}$ While we do not know how to connect their results to ours, it seems that there is one common theme: that high-energy terms find their way to affect the physics at intermediate temperatures.

The chances to find a constrained entropic critical point improve if one adds nearest-neighbor, next-nearest neighbor, etc., couplings-i.e., in extended versions of the Hubbard model - at some commensurate fillings. For example, the presence of very strong nearest-neighbor interactions at $1 / 4$ filling leads to a classical checkerboard configuration, where one of the two sublattices of the bipartite square lattice is filled and the other empty. If one now starts changing the doping away from 1/4 filling, extra holes or particles will tend to cluster in stripes. ${ }^{36-42}$ Other longer-range couplings that commute with the constraint could lead to charge ordering, perhaps in the form of static stripes or other patterns at low temperatures. Now, at high temperatures, when charge order is destroyed, these stripes meander and fluctuate, and 
the system could be in an entropic critical regime. Certainly, these are speculative thoughts, in contrast to the concrete cases of the constrained quantum Heisenberg and transverse field Ising models that we presented as examples of constrained entropic criticality. But the discussion suggests how it is not implausible that a constrained entropic critical point may play a role in the physics of the extended Hubbard model near commensurate fillings.

\section{ACKNOWLEDGMENTS}

We would like to thank S. Chakravarty, V. Dotsenko, and M. Picco, for their insightful comments. We are particularly indebted to E. Fradkin for several useful discussions and for his careful reading of the manuscript. This work is supported in part by the NSF Grant Nos. DMR-0305482 and DMR0403997 (C.C. and C.C.)
${ }^{1}$ E. Dagotto, Science 309, 257 (2005), and references therein.

${ }^{2}$ A. Kopp and S. Chakravarty, Nat. Phys. 1, 53 (2005).

${ }^{3}$ C. M. Varma, Phys. Rev. B 55, 14554 (1997).

${ }^{4}$ S. Sachdev, Rev. Mod. Phys. 75, 913 (2003).

${ }^{5}$ S. A. Kivelson, I. P. Bindloss, E. Fradkin, V. Oganesyan, J. M. Tranquada, A. Kapitulnik, and C. Howald, Rev. Mod. Phys. 75, 1201 (2003).

${ }^{6}$ S. Chakravarty, Phys. Rev. B 66, 224505 (2002).

${ }^{7}$ J. Hubbard, Proc. R. Soc. London, Ser. A 276, 238 (1963).

${ }^{8}$ G. Müller, Phys. Rev. B 26, 1311 (1982).

${ }^{9}$ C. Castelnovo, C. Chamon, C. Mudry, and P. Pujol, Phys. Rev. B 72, 104405 (2005).

${ }^{10}$ R. J. Elliott, P. Pfeuty, and C. Wood, Phys. Rev. Lett. 25, 443 (1970).

${ }^{11}$ P. W. Anderson, in Solid State Physics, edited by F. Seitz and D. Turnbull (Academic, New York, 1963), Vol. 14, p. 99.

${ }^{12}$ V. J. Emery, Phys. Rev. B 14, 2989 (1976).

${ }^{13}$ K. A. Chao, J. Spalek, and A. M. Oles, Phys. Lett. 64A, 163 (1977); J. Phys. C 10, L271 (1977).

${ }^{14}$ R. J. Baxter, J. Math. Phys. 11, 784 (1970).

${ }^{15}$ P. W. Kasteleyn, J. Math. Phys. 4, 287 (1963).

${ }^{16}$ N. Read (unpublished); J. Kondev and C. L. Henley, Nucl. Phys. B 464, 540 (1996); J. Kondev, J. de Gier, and B. Nienhuis, J. Phys. A 29, 6489 (1996).

${ }^{17}$ H. W. J. Blöte, J. L. Cardy, and M. P. Nightingale, Phys. Rev. Lett. 56, 742 (1986).

${ }^{18}$ I. Affleck, Phys. Rev. Lett. 56, 746 (1986).

${ }^{19}$ J. E. Moore and D.-H. Lee, Phys. Rev. B 69, 104511 (2004).

${ }^{20}$ C. Castelnovo, P. Pujol, and C. Chamon, Phys. Rev. B 69, 104529 (2004).

${ }^{21}$ E. N. M. Cirillo, G. Gonnella, and A. Pelizzola, Phys. Rev. E 53, 1479 (1996).

${ }^{22}$ E. Fradkin, D. A. Huse, R. Moessner, V. Oganesyan, and S. L. Sondhi, Phys. Rev. B 69, 224415 (2004).

${ }^{23}$ E. Fradkin, Field Theories of Condensed Matter Systems (Addison-Wesley, Redwood City, CA, 1991); see Chap. 6.
${ }^{24}$ D. S. Rokhsar and S. A. Kivelson, Phys. Rev. Lett. 61, 2376 (1988).

${ }^{25}$ A. W. Sandvik and R. Moessner, cond-mat/0507277, Phys. Rev. B (to be published); A. W. Sandvik, cond-mat/0312097 (unpublished).

${ }^{26}$ P. Fendley, R. Moessner, and S. L. Sondhi, Phys. Rev. B 66, 214513 (2002).

${ }^{27}$ F. Alet, J. L. Jacobsen, G. Misguich, V. Pasquier, F. Mila, and M. Troyer, Phys. Rev. Lett. 94, 235702 (2005).

${ }^{28}$ O. F. Syljuåsen, cond-mat/0512579 (unpublished).

${ }^{29}$ K. Penc, N. Shannon, and H. Shiba, Phys. Rev. Lett. 93, 197203 (2004).

${ }^{30}$ D. L. Bergman, R. Shindou, G. A. Fiete, and L. Balents, Phys. Rev. Lett. 96, 097207 (2006).

${ }^{31}$ D. L. Bergman, G. A. Fiete, and L. Balents, cond-mat/0511176 (unpublished).

${ }^{32}$ S. Chakravarty, R. B. Laughlin, D. K. Morr, and C. Nayak, Phys. Rev. B 63, 094503 (2001).

${ }^{33}$ O. F. Syljuåsen and S. Chakravarty, cond-mat/0509624 (unpublished).

${ }^{34}$ P. Phillips, D. Galanakis, and T. D. Stanescu, Phys. Rev. Lett. 93, 267004 (2004); T.-P. Choy and P. Phillips, ibid. 95, 196405 (2005).

${ }^{35}$ P. Phillips and C. Chamon, Phys. Rev. Lett. 95, 107002 (2005).

${ }^{36}$ U. Löw, V. J. Emery, K. Fabricius, and S. A. Kivelson, Phys. Rev. Lett. 72, 1918 (1994).

${ }^{37}$ F. Mila, Phys. Rev. B 49, R14047 (1994).

${ }^{38}$ C. L. Henley and N.-G. Zhang, Phys. Rev. B 63, 233107 (2001).

${ }^{39}$ R. H. McKenzie, J. Merino, J. B. Marston, and O. P. Sushkov, Phys. Rev. B 64, 085109 (2001).

${ }^{40}$ E. Arrigoni, A. P. Harju, W. Hanke, B. Brendel, and S. A. Kivelson, Phys. Rev. B 65, 134503 (2002).

${ }^{41}$ N. G. Zhang and C. L. Henley, Phys. Rev. B 68, 014506 (2003).

${ }^{42}$ E. Arrigoni, E. Fradkin, and S. A. Kivelson, Phys. Rev. B 69, 214519 (2004). 